\title{
How the stability of a folded protein depends on interfacial water properties and residue-residue interactions
}

\author{
Valentino Bianco ${ }^{\mathrm{a},{ }^{*}}$, Neus Pagès Gelabert ${ }^{\mathrm{b}}$, Ivan Coluzza ${ }^{\mathrm{a}}$, Giancarlo Franzese $^{\mathrm{b}, \mathrm{c},{ }^{*}}$ \\ ${ }^{a}$ Universität Wien, Sensengasse 8/10, Vienna 1090, Austria \\ b Secció de Física Estadistica i Interdisciplinària-Departament de Física de la Matèria Condensada, Facultat de Física Universitat de Barcelona, Martí i Franquès 1, Barcelona \\ 08028, Spain \\ ${ }^{\mathrm{c}}$ Institute of Nanoscience and Nanotechnology (IN2UB), Universitat de Barcelona, Av. Joan XXIII S/N, Barcelona 08028, Spain
}

\section{A R T I C L E I N F O}

Article history:

Received 11 April 2017

Received in revised form 4 August 2017

Accepted 6 August 2017

Available online $\mathrm{xxx}$

Keywords:

Protein folding

Cold denaturation

Pressure denaturation

Interfacial water

Explicit water

Biological physics

\begin{abstract}
A B S T R A C T
Proteins tend to adopt a single or a reduced ensemble of configurations at natural conditions [1], but changes in temperature $T$ and pressure $P$ induce their unfolding. Therefore for each protein there is a stability region (SR) in the $T-P$ thermodynamic plane outside which the biomolecule is denaturated. It is known that the extension and shape of the SR depend on i) the specific protein residue-residue interactions in the native state of the amino acids sequence and ii) the water properties at the hydration interface. Here we analyze by Monte Carlo simulations the different coarse-grained protein models in explicit water how changes in i) and ii) affect the SR. We show that the solvent properties ii) are essential to rationalize the SR shape at low $T$ and high $P$ and that our findings are robust with respect to parameter changes and with respect to different protein models, representative of the ordered and disordered proteins. These results can help in developing new strategies for the design of novel synthetic biopolymers.
\end{abstract}

(C) 2017 .

\section{Introduction}

The capability of the single components to independently organize in pattern and structures without an external action fulfills a crucial role in the supramolecular organization and assembling of the biological matter [2,3]. To cite some examples, self-assembly is observed in bio-molecules [4], in DNA and chromosomes [5-8], in lipid membranes $[9,10]$, in the cytoskeleton [11], in cells and tissues $[12,13]$, in virus and bacteria $[14,15]$, and in proteins $[16,17]$. In particular, the protein folding represents one of the most challenging and elusive biochemical processes where a chain of amino acids organizes itself into a unique native and folded structure $[18,19]$. The protein folding is a spontaneous process driven by intra-molecular (residue-residue) van der Walls interactions and hydrogen bonds which overcome the conformational entropy. It depends also on the presence of co-factors as the chaperones [20] and, in particular, the properties of the solvent, i.e. water [21], and the co-solutes [22] that regulate the $\mathrm{pH}$ level and the salt concentration, for example.

Although water has no influence on the primary structure (the protein sequence), it affects the protein in all the other level of organization [23-25]. Indeed, i) water forms H-bonds with the polar/charged residues of the side chains, influencing the adoption of secondary structures like alpha helices or beta sheets which expose the most hy-

\footnotetext{
* Corresponding authors.

Email addresses: valentino.bianco@univie.ac.at (V. Bianco); gfranzese@ub.edu (G. Franzese)
}

drophilic residues to water; ii) the hydrophobic effect drives the collapse of the protein core and stabilizes the tertiary protein structure; iii) water induces the aggregation of proteins since they usually present hydrophobic regions on their surface (quaternary structure).

The stability of a protein, i.e. its capability to keep the folded conformation, is usually reduced by factors which destabilize H-bonding and other forces that contribute to secondary and tertiary protein structure, as, for example, crowding effects and variations of $\mathrm{pH}$ or ionic strength. In particular, experiments have clearly documented that proteins maintain their native structure in a limited range of temperatures $T$ and pressures $P$ [26-41] showing an elliptic-like stability region (SR) in the $T-P$ plane, as accounted by a Hawley's theory [42]. Outside its SR a protein unfolds, with a consequent loss of its tertiary structure and functionality.

At high $T$ the protein unfolding is due to the thermal fluctuations which disrupt the protein structure. Open protein conformations increases the entropy $S$ minimizing the global Gibbs free energy $G \equiv H-T S$, where $H$ is the total enthalpy. Upon cooling, if the nucleation of water is avoided, some proteins cold-denaturate $[27,29,34,36,43-46]$. Usually such a phenomenon is observed below the melting line of water, although in some cases cold denaturation occurs above the $0^{\circ} \mathrm{C}$, as in the case of the yeast frataxin [36]. Protein denaturation is observed, or predicted, also upon pressurization $[26,28,35,41,47]$. A possible explanation of the high- $P$ unfolding is the loss of internal cavities, sometimes presents in the folded states of proteins [48]. Denaturation at negative $P$ has been experimentally observed [49] and simulated recently $[21,49,50]$. Pressure denatura- 
tion is usually observed for $100 \mathrm{MPa} \lesssim P \lesssim 600 \mathrm{MPa}$, and rarely at higher $P$ unless the tertiary structure is engineered with stronger covalent bonds [33]. Cold- and $P$-denaturation of proteins have been related to the equilibrium properties of the hydration water [21,51-60]. However, the interpretations of the mechanism is still largely debated [47,48,61-71].

Here we investigate by Monte Carlo simulations of different coarse-grained protein models in explicit water how the SR is affected by changes in i) the specific protein residue-residue interactions in the native state of the amino acids sequence and ii) the solvent properties at the hydration interface, focusing on water energy and density fluctuations. In particular, after introducing the model and the numerical method in Section 2, we study in a broad range of $T$ and $P$ how the conformational space of proteins depends on the model's parameters for the hydration water in Section 3.1 and how it depends on the residue-residue interactions in Section 3.2. Next, we discuss the possible relevance of our results in the framework of protein design in Section 4 and, finally, we present our concluding remarks in Section 5.

\section{Models and methods}

The extensive exploration with atomistic models of protein conformations in explicit solvent at different thermodynamic conditions, including extreme low $T$ and high $P$, is a very demanding analysis. To overcome this limitation, we adopt a coarse-grain model for protein-water interaction based on A) the many-body water model $[21,60,67,72-80]$, combined with B) a lattice representation of the protein.

The many-body water model has been proven to reproduce-in at least qualitative way-the thermodynamic [72,80] and dynamic [78] behavior of water, the properties of water in confinement $[73,74,77,79]$ and at the inorganic interfaces [67]. Its recent combination with the lattice representation of the protein has given a novel insight into the water-protein interplay [21,60,75,76,81].

As we will describe later, for the protein we consider a model that, in its general formulation as polar protein, follows the so-called "Go-models", a common approach in protein folding. In their seminal paper Go and Taketomi [82] employed non-transferable potentials tailored to the native structure. The interactions were designed to have a sharp minimum only at the native residue-residue distance, guaranteeing that the energy minimum is reached only by the native structure. The Go-proteins thus successfully fold, and have a smooth free-energy landscape with a single global minimum in the native structure [83]. Hence, Go-models are equivalent to having an infinite variety of pair interactions among the residues (alphabet $\mathscr{A}$ ), such that each amino acid interacts selectively with a subset of residues defined by the distances in the native configuration. If the size of the alphabet is reduced, the construction of folding proteins requires an optimization step of amino acid sequence along the chain $[63,84,85]$; for this reason these methods are often referred to as "protein design". Comparing designed proteins with Go-proteins, Coluzza recently shown that, close to the folded state, Go and designed proteins behave in a very similar manner [86]. Since we are interested in measuring the stability regions defined by the environmental condition at which the trial protein is at least $90 \%$ folded, Go-models are an appropriate protein representation, and, at this stage, we do not require to perform the laborious work of protein design to obtain general results. We will discuss later the possibility to extend our model to the case of a limited alphabet $\mathscr{A}$ of residues (20 amino acids).

\subsection{The bulk many-body water model}

We consider the coarse-grain many-body bulk water at constant $P$, constant $T$ and constant number $N^{(\mathrm{b})}$ of water molecules, while the total volume $V^{(b)}$ occupied by water is a function of $P$ and $T$. Because in the following we will consider the model with water at the hydration protein interface and (bulk) water away from the interface, for the sake of clarity here we introduce the notation with a superscript (b) for quantities that refer to the bulk.

We replace the coordinates and orientations of the water molecules by a continuous density field and discrete bonding variables, respectively. The density field is defined based on a partition of the available volume $V^{(\mathrm{b})}$ into a fixed number $N_{0}=N^{(\mathrm{b})}$ of cells, each with volume $v^{(\mathrm{b})} \equiv V^{(\mathrm{b})} / N^{(\mathrm{b})} \geq v_{0}$, where $v_{0} \equiv r_{0}^{3}$ is the water excluded volume with $r_{0} \equiv 2.9 \AA$ (water van der Waals diameter). For the sake of simplicity we assume that, when the water molecules are not forming hydrogen bonds (HBs), the (dimensionless) density is homogeneous in each cell and equal to $\rho^{(\mathrm{b})} \equiv v_{0} / v^{(\mathrm{b})}$. As we will discuss later, the density is, instead, locally inhomogeneous when water molecules form HBs. Specifically, the density depends on the number of HBs, therefore $\rho^{(\mathrm{b})}$ only represents the average bulk density.

The Hamiltonian of the bulk water is

$$
\mathscr{H}^{(\mathrm{b})} \equiv \sum_{i j} U\left(r_{i j}\right)-J N_{\mathrm{HB}}^{(\mathrm{b})}-J_{\sigma} N_{\mathrm{coop}}^{(\mathrm{b})}
$$

The first term represents the isotropic part of the water-water interaction and accounts for the van der Waals interaction [87]. It is modeled with a Lennad-Jones potential

$$
\sum_{i j} U\left(r_{i j}\right) \equiv 4 \epsilon \sum_{i j}\left[\left(\frac{r_{0}}{r_{i j}}\right)^{12}-\left({\frac{r_{0}}{r_{i j}}}^{6}\right)\right]
$$

where $\epsilon \equiv 5.8 \mathrm{~kJ} / \mathrm{mol}$ and the sum runs over all the water molecules $i$ and $j$ at $\mathrm{O}-\mathrm{O}$ distance $r_{i j}$ calculated as the distance between the centers of the two cells $i$ and $j$ where the molecules belong. We assume a hard-core exclusion $U(r) \equiv \infty$ for $r<r_{0}$ and a cutoff for $r>r_{c} \equiv 6 r_{0}$.

The second term in Eq. (1) represents the directional (covalent) component of the HB, where

$$
N_{\mathrm{HB}}^{(\mathrm{b})} \equiv \sum_{\langle i j\rangle} n_{i} n_{j} \delta_{\sigma_{i j}, \sigma_{j i}}
$$

is the number of bulk HBs and the sum runs over neighbor cells occupied by water molecules. Here we introduce the label $n_{i}=1$ if the cell $i$ has a water density $\rho^{(\mathrm{b})}>0.5$ and $n_{i}=0$ otherwise. In the homogeneous bulk this condition guarantees that two water molecules can form a $\mathrm{HB}$ only if their relative distance is $r<2^{1 / 3} r_{0} \equiv 3.66 \AA$, corresponding to the range of a water's first coordination shell as determined from the $\mathrm{O}-\mathrm{O}$ radial distribution function from 220 to $673 \mathrm{~K}$ and at pressures up to $400 \mathrm{MPa}$ [88].

The variable $\sigma_{i j}=1, \ldots, q$ in Eq. (3) is the bonding index of the water molecule in cell $i$ with respect to the neighbor molecule in cell $j$ and $\delta_{a b}=1$ if $a=b$, or 0 otherwise, is a Kronecker delta function. Each water molecule has as many bonding variables as neighbor cells, but can form only up to four HBs. Therefore, if the molecule has more than four neighbors, e.g., in a cubic lattice partition of $V^{(b)}$, 
an additional condition must be applied to limit to four the HBs participated by each molecule.

The parameter $q$ in the definition of $\sigma_{i j}$ is determined by the entropy decrease associated to the formation of each HB. Each HB is unbroken if the hydrogen atom $\mathrm{H}$ is in a range of $\left[-30^{\circ}, 30^{\circ}\right]$ with respect to the $\mathrm{O}-\mathrm{O}$ axes [89]. Hence, only $1 / 6$ of the entire range of values $\left[0,360^{\circ}\right]$ for the $\widehat{\mathrm{OOH}}$ angle is associated to a bonded state. Therefore, in the zero-order approximation of considering each HB independent, a molecule that has $4-n \mathrm{HBs}$, with $n=1, \ldots 4$, has an orientational entropy that is $S_{n}^{\mathrm{o}} / k_{B} \equiv n \ln 6$ above that of a fully bonded molecule with $S_{0}^{\mathrm{o}} / k_{B}$ $\equiv 0$, where $k_{B}$ is the Boltzmann constant. As a consequence, the choice $q=6$ accounts correctly for the entropy variation due to HB formation and breaking given the standard definition of $\mathrm{HB}$.

The third term in Eq. (1) is associated to the cooperativity of the HBs due to the quantum many-body interactions [72,90]. Indeed, the formation of a new $\mathrm{HB}$ affects the electron probability distribution around the molecule favoring the formation of the following $\mathrm{HB}$ in a local tetrahedral structure [91]. We assume that the energy gain due to this effect is proportional to the number of cooperative HBs in the system

$$
N_{\text {coop }} \equiv \sum_{i} n_{i} \sum_{(l, k)_{i}} \delta_{\sigma_{i k}, \sigma_{i l}}
$$

where $n_{i}$ assures that we include this term only for liquid water. With this definition and with the choice $J_{\sigma} / 4 \epsilon \ll J$ the term mimics a many-body interactions among the HBs participated by the same molecule. Indeed, the condition $J_{\sigma} / 4 \epsilon \ll J$ guarantees that the interaction takes place only when the water molecule $i$ is forming several HBs. The inner sum is over $(l, k)_{i}$, indicating each of the six different pairs of the four indices $\sigma_{i j}$ of the molecule $i$.

The formation of HBs leads to an open network of molecules, giving rise to a lower density state. We include this effect into the model assuming that for each HB the volume $V^{(\mathrm{b})}$ increases of $v_{\mathrm{HB}}{ }^{(\mathrm{b})} / v_{0}=0.5$. This value is the average volume increase between high-density ices VI and VIII and low-density (tetrahedral) ice Ih. As a consequence, the average bulk density is

$$
\rho^{(\mathrm{b})} \equiv \frac{N v_{0}}{V^{(\mathrm{b})}+N_{\mathrm{HB}}^{(\mathrm{b})} v_{\mathrm{HB}}^{(\mathrm{b})}}
$$

We assume that the HBs do not affect the distance $r$ between first neighbor molecules, consistent with experiments [91]. Hence, the water-water distances $r$ is calculated only from $V^{(\mathrm{b})}$.

As discussed in Ref. [21] a good choice for the parameters that accounts for the ions in a protein solution is $\epsilon=5.8 \mathrm{~kJ} / \mathrm{mol}, J / 4 \epsilon=0.3$ and $J_{\sigma} / 4 \epsilon=0.05$ that give an average HB energy $\sim 20 \mathrm{~kJ} / \mathrm{mol}$. In the following we consider two protein models, a simpler one used to understand the molecular mechanisms through which water contributes to the unfolding, and a more detailed model which includes the effect of polarization. For the sake of simplicity, we present here the result for a system in two dimension. Preliminary results for the model in three dimensions of both bulk water [80] and protein folding show results that are qualitatively similar to those presented here.

\subsection{Hydrophobic protein model}

The protein is modeled as a self-avoiding lattice polymer, embedded into the cell partition of the system. Despite its simplicity, lattice protein models are still widely used in the contest of protein folding $[21,52,53,59,71,92-94]$ because of their versatility and the possibility to develop coarse-grained theories and simulations for them. Each protein residue (polymer bead) occupies one cell. In the present study, we do not consider the presence of cavities into the protein structure.

To simplify the discussion in this first part of the work, we assume that (i) there is no residue-residue interaction, (ii) the residue-water interaction vanishes, unless otherwise specified and (iii) all the residues are hydrophobic. This implies that the protein has multiple ground states, all with the same maximum number $n_{\max }$ of residue-residue contacts. As shown by Bianco and Franzese [21], the results hold also when the hypothesis (i), (ii) and (iii) are released, as we will discuss in the following.

Our stating hypothesis is that the protein interface affects the water-water properties in the hydration shell, here defined as the layer of first neighbor water molecules in contact with the protein (Fig. 1). There are many numerical and experimental evidences supporting this hypothesis. In particular, it has been shown that the water-water HBs in the protein hydration shell are more stable and more correlated with respect to the bulk HBs by using theoretical calculations [95], experiments $[96,98,99]$ and atomistic simulations $[97,99,100]$. We account for this by replacing $J$ of Eq. (1) with $J_{\Phi}>J$ for the water-water HBs at the hydrophobic $(\Phi)$ interface. Another possibility, discussed later, would be to consider that the cooperative interaction $J_{\sigma, \Phi}$ at the $\Phi$-interface, directly related to the tetrahedral order of the water molecules, is stronger with respect to the bulk. This case would be consistent with the assumption that water forms ice-like cages around $\Phi$-residues [101]. Both choices, according to Muller discussion [102], would ensure the water enthalpy compensation during the cold-denaturation [60].

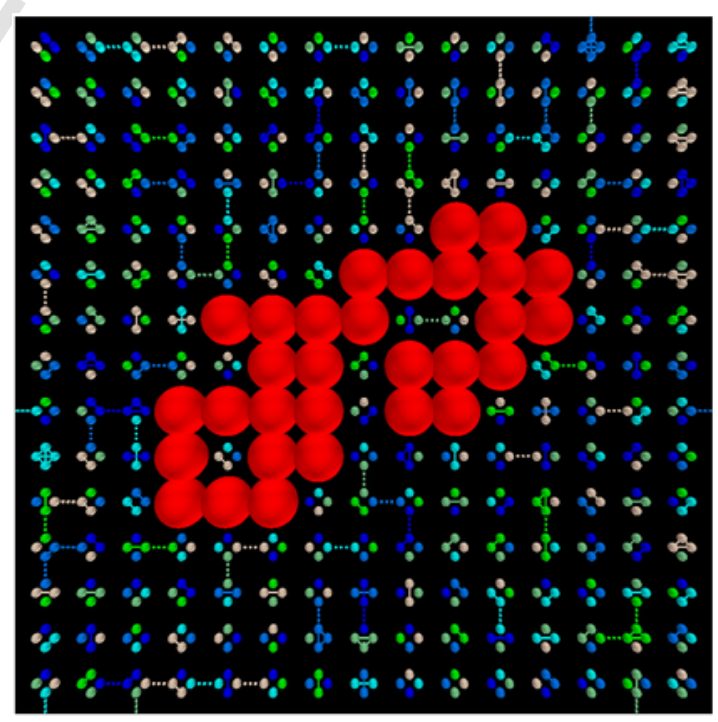

Fig. 1. Scheme of the water-protein coarse grain model. The protein is represented with red spheres. Each water molecule is represented through its 4 bonding indexes $\sigma$, with different colors associated to the value $1 \ldots q$ assumed by $\sigma$. Directional HB are represented with dotted lines joining two water molecules. Cooperative bonds are represented with continuous lines connecting the $\sigma$ indices inside a molecule. 
At the $\Phi$-interface, besides the stronger/stabler water-water HB, we consider also the larger density fluctuations with respect to the bulk. These larger densities fluctuations have been observed in extensive coarse-grained molecular dynamics simulations including explicit solvation [65,103] and extensive atomistic molecular simulations [97] of hydrated $\Phi$-solutes.

Although it is still a matter of debate if, at ambient conditions, the average density of water at the $\Phi$-interface is larger or smaller with respect to the average bulk water density [104-108], there are evidences showing that such density fluctuations reduce upon pressurization $[65,97,109,110]$. We include this effect in the model by assuming that the volume change $v_{\mathrm{HB}}{ }^{(\Phi)}$ associated to the HB formation in the $\Phi$ hydration shell can be expanded as a series function of $P$

$$
v_{\mathrm{HB}}^{(\Phi)} / v_{\mathrm{HB}, 0}^{(\Phi)} \equiv 1-k_{1} P-k_{2} P^{2}-k_{3} P^{3}+O\left(P^{4}\right)
$$

where $v_{\mathrm{HB}, 0}{ }^{(\Phi)}$ is the value of the change when $P=0$. Here the coefficients $k_{1}, k_{2}$ and $k_{3}$ are such that $\partial v_{\mathrm{HB}}{ }^{(\Phi)} / \partial P$ is always negative. As first approximation, we study the linear case, with $k_{i}=0 \forall i>1$. We discuss later how the protein stability is affected by considering the quadratic terms in Eq. (6). Our initial choice implies that we can study the system only when $P<1 / k_{1}$. As we will discuss in the next section, this condition does not limit the validity of our results. The total volume $V$ of the system is, therefore,

$$
V \equiv N v_{0}+N_{\mathrm{HB}}^{(\mathrm{b})} v_{\mathrm{HB}}^{(\mathrm{b})}+N_{\mathrm{HB}}^{(\Phi)} v_{\mathrm{HB}}^{(\Phi)}
$$

where $N_{\mathrm{HB}}^{(\Phi)}$ is the number of HBs in the $\Phi$ shell.

\subsection{Polar protein model}

In order to account for the effect of the hydrophilic residues on the water-water hydrogen bonding in the hydration shell, we consider also the case in which the protein is modeled as a heteropolymer composed of hydrophobic $(\Phi)$ and hydrophilic $(\zeta)$ residues. In this case is worth introducing residue-residues interactions that lead to a specific folded (native) state for the protein.

We fix the native state by defining the interaction matrix $A_{i, j} \equiv \epsilon_{\mathrm{rr}}$ if residues $i$ and $j$ are n.n. in the native state, 0 otherwise. To simplify our model we set all the residues in contact with water in the native state as hydrophilic, and all those buried into the protein core as hydrophobic. The water interaction with $\Phi$ - and $\zeta$-residues is given by the parameters $\epsilon_{\mathrm{w}, \Phi}$ and $\epsilon_{\mathrm{w}, \zeta}$ respectively, where we assume $\epsilon_{\mathrm{w}, \Phi}<J$ and $\epsilon_{\mathrm{w}, \zeta}>J$.

The polar $\zeta$ residues interfere with the formation of HB of the surrounding molecules, disrupting the tetrahedral order and distorting the HB network. Thus we assume that each $\zeta$ residue has a preassigned bonding state $q^{(\zeta)}=1, \ldots, q$, different and random for each $\zeta$ residue. In this way, a water molecule $i$ can form a HB with a $\zeta$ residue, located in the direction $j$, only if $\sigma_{i, j}=q^{(\zeta)}$.

In the polar potein model, the formation of water-water HBs in the hydration shell is described by the parameters i) $J_{\Phi}$ and $J_{\sigma, \Phi}$ (directional and cooperative components of the $\mathrm{HB}$ ) if both molecules hydrate two $\Phi$-residues; ii) $J_{\zeta}$ and $J_{\sigma, \zeta}$ if both molecules hydrate two $\zeta$-residues; iii) $J_{\Phi, \zeta} \equiv\left(J_{\Phi}+J_{\zeta}\right) / 2$ and $J_{\sigma, \Phi, \zeta} \equiv\left(J_{\sigma, \Phi}+J_{\sigma, \zeta}\right) / 2$ if the two water molecules are in contact one with a $\Phi$-residue and another with a $\zeta$-residue, forming a $\Phi$ - $\zeta$-interface. Accordingly, the volume associated to the formation of $\mathrm{HB}$ in the hydration shell is $v_{\mathrm{HB}}{ }^{(\Phi)}, v_{\mathrm{HB}}{ }^{(\zeta)}$ and $v_{\mathrm{HB}}{ }^{(\Phi, \zeta)}$. Then, we assume that $v_{\mathrm{HB}}{ }^{(\Phi)}$ changes with $P$ following the Eq. (6). Due to the condition $\epsilon_{\mathrm{w}, \zeta}>J$, we assume that the density fluc- tuations near a $\zeta$-residue are comparable, or smaller, than those in bulk water, therefore we set $v_{\mathrm{HB}}{ }^{(\zeta)}=v_{\mathrm{HB}}{ }^{(b)}$. Finally, we define $v_{\mathrm{HB}}^{(\Phi, \zeta)} \equiv\left(v_{\mathrm{HB}}^{(\Phi)}+v_{\mathrm{HB}}^{(\zeta)}\right) / 2$.

\subsection{Simulations' details}

We study proteins of 30 residues with Monte Carlo simulations in the isobaric-isothermal ensemble, i.e. with constant $P$, constant $T$ and constant number of particles. Along the simulation we calculate the average number of residue-residue contacts to estimate the protein compactness, sampling $\sim 10^{5}$ independent protein conformations for each thermodynamic state point. For the hydrophobic protein model, we assume that the protein is folded if the average number of residue-residue contacts is $n_{\mathrm{rr}} \geq 50 \% n_{\max }$, while for the polar protein model, having a unique folded state, we fix the threshold at $n_{\mathrm{rr}} \geq 90 \%$ $n_{\text {max }}$.

For the sake of simplicity, we consider our model in two dimensions. Although this geometry could appear as not relevant for experimental cases, our preliminary results for the three dimensional system show no qualitative difference with the case presented here. We understand this finding as a consequence of the peculiar property of bulk water of having, on average, not more than four neighbors. This coordination number is preserved if we consider a square partition of a two dimensional system. Differences between the two dimensional and the three dimensional models could arise from the larger entropy in higher dimensions for the protein, however our preliminary results in $3 \mathrm{D}$ show that they can be accounted for by tuning the model parameters.

\section{Results and discussion}

\subsection{Results for the hydrophobic protein model}

Bianco and Franzese show [21] that the hydrophobic protein model, with parameters $k_{1}=v_{0} / 4 \epsilon$ (and $k_{2}=k_{3}=0$ ), $v_{\mathrm{HB}, 0}{ }^{(\Phi)} / v_{0}=v_{\mathrm{HB}}{ }^{(\mathrm{b})} / v_{0}=0.5$ and $J_{\Phi} / 4 \epsilon=0.55, J_{\sigma, \Phi}=J_{\sigma}$, has a SR that is elliptic in the $T-P$ plane. This finding is consistent with the predictions of the Hawley theory $[31,42]$ accounting for the thermal, cold and pressure denaturation (Fig. 2).

They find that at high $T$ the large entropy associated to open protein conformations keeps the protein unfolded. By isobaric decrease of $T$, the energy cost of an extended water-protein interface can no longer be balanced by the entropy gain of the unfolded protein, and the protein folds to minimize the number of hydrated $\Phi$-residues, as expected.

By further decreasing of $T$ at constant $P$, the number of water-water HBs increases both in bulk and at the protein interface. At low-enough $T$, the larger stability, i.e., larger energy gain, of the HBs at the $\Phi$-interface drives the cold denaturation of the protein.

Upon isothermal increase of $P$, the enthalpy of the system increases for the increasing $P V$ term. Therefore, a mechanism that reduces $V$ would reduce the total enthalpy. Here the mechanism is provided by the water compressibility that is larger at the $\Phi$-interface than in bulk. Therefore, the larger water density at the protein interface drives the unfolding, which leads to a larger $\Phi$-interface and enthalpy gain.

Finally, when the system is under tension, i.e., at $P<0$, the total enthalpy is minimized when $V$ in Eq. (7) is maximized. However, the increase of average separation between water molecules breaks the HBs. In particular, bulk HBs break more than those at the $\Phi$-interface because the first are weaker than the latter. Hence, $N_{\mathrm{HB}}{ }^{(b)}$ vanishes when $N_{\mathrm{HB}}{ }^{(\Phi)}>0$. As a consequence, the maximization of $V$ is 


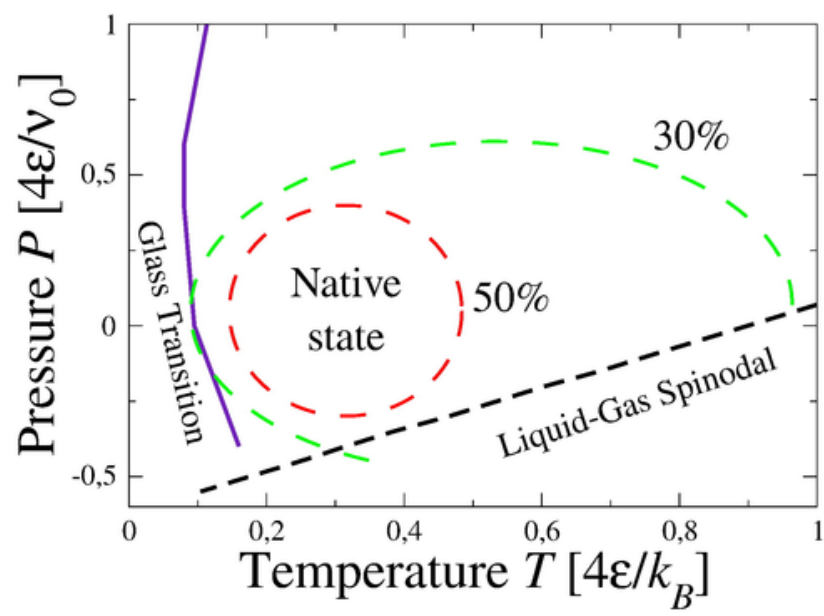

Fig. 2. Stability region for a coarse-grained proteins made of 30 hydrophobic residues. The dotted green line delimits the region within which the protein makes at least $30 \%$ of its maximum number of contact points, i.e., $n_{\mathrm{rr}} / n_{\max } \geq 0$.3. Inside this region, the dotted red line delimits the set of states for which $n_{\mathrm{rr}} / n_{\max } \geq 0.5$, that by definition correspond to the native state of the folded protein. The lower straight (black) dotted line represents the limit of stability (spinodal) of the liquid water with respect to the gas. The left-most (violet) solid line marks the limit below which water forms a glass state. Adapted from Ref. [21].

achieved by maximizing $N_{\mathrm{HB}}{ }^{(\Phi)}$, i.e., by exposing the maximum number of $\Phi$-residues, leading to the protein denaturation under tension.

Once it is clear that the model can reproduce the protein SR, allowing us to understand the driving mechanism for the denaturation at different thermodynamic conditions, it is insightful to study how the SR depends on the model parameters. Therefore, in the following of this work we show our new calculations about the effect of varying one by one the model parameters.

\subsubsection{Varying the water-water $H B$ directional component $J_{\Phi}$ at the $\Phi$-interface}

Changing the (covalent) strength $J_{\Phi}$ of the interfacial HB has a drastic effect on the SR. As discussed above, having $J_{\Phi} / J>1$, as in the reference case, drives the cold unfolding as a consequence of the larger gain of HB energy near the $\Phi$-interface. Instead, by setting $J_{\Phi} / J$ $<1$ (Fig. 3a) the folded protein becomes more stable at low $T$ than in the reference case, because there is a larger energy gain in forming as many bulk $\mathrm{HB}$ as possible, i.e., in reducing the number of those near $\Phi$-residues. Hence, there is a larger free-energy gain in reducing the exposed $\Phi$-interface with respect to the reference case.

As a matter of fact, with our choice $J_{\Phi} / 4 \epsilon=0.20$, we find cold denaturation only for $P<0$. This is a consequence of the fact that the free energy has a term with $N_{\mathrm{HB}}{ }^{(\Phi)}$ multiplying $\left(-J_{\Phi}+P v_{\mathrm{HB}}-P^{2} v_{\mathrm{HB}} v_{0} / 4 \epsilon\right)$, hence for $P<0$ the free energy decreases if $N_{\mathrm{HB}}{ }^{(\Phi)}$ increases, even for a vanishing $J_{\Phi}$. The negative slope of the cold denaturation line at $P<0$ (Fig. 3a for $70 \%$ curve) is because the larger the $|P|$, the larger is the term proportional to $N_{\mathrm{HB}}^{(\Phi)}$ in the free-energy balance.

Reducing $J_{\Phi}$ makes the folded protein more stable also at high $T$, because the entropy term overcomes the energy term at $T$ lower than in the reference case. A similar observation holds also at high $P$, because a reduced $J_{\Phi}$ implies a decrease in $N_{\mathrm{HB}}{ }^{(\Phi)}$, hence a decrease in enthalpy gain associated to the exposure of the $\Phi$-interface.

On the other hand, the larger the $|P|$, the more negative is the quadratic $P$-dependent coefficient that, as mentioned above, multiplies $N_{\mathrm{HB}}{ }^{(\Phi)}$ in the free energy, and the larger is the free-energy gain in exposing the $\Phi$-interface at high $T$. Hence, the hot-denaturation curve in (a)



(b)



Fig. 3. Effect on the SR of changing the water-water HB directional component $J_{\Phi}$ at the $\Phi$-interface. In both panels symbols with continuous lines delimit the regions with $30 \%$ (green), $40 \%$ (turquoise), $50 \%$ (red) and 70\% (blue) of the protein folded. Dashed lines (with the same color code as for continuous lines) are for the reference system in Fig. 2 (Table 1) with $J_{\Phi} / 4 \epsilon=0.55$. All lines are guide for eyes. (a) For $J_{\Phi} / 4 \epsilon=0.20$, smaller than the reference value, the SR expands to lower $T$ and $P$ and to higher $T$ and $P$. (b) For $J_{\Phi} / 4 \epsilon=0.75$, greater than the reference value, the SR shrinks.

the $P$ - $T$ plane has a negative slope for $P>0$ and a positive slope for $P$ $<0$. As a consequence, the ellipsis describing the SR (Fig. 3a for 50\% curve) becomes more elongated than in the reference case with a negatively-sloped major axis and an eccentricity that grows toward 1.

On the contrary, for increasing $J_{\Phi}$ the SR is lost, due to the energetic gain associated to wetting the entire $\Phi$-interface of the protein (Fig. 3b). The $P$-dependence of the contour lines is the same as that discussed for the case with $J_{\Phi} / J<1$, hence they keep the shape but shrink.

\subsubsection{Varying the water compressibility factor $k_{1}$ at the $\Phi$-interface}

Decreasing the water compressibility factor $k_{1}$ leads to a stretching of the SR along the $P$ direction and a rotation of the ellipse axes in a such a way that the main axis increases its negative slope in the $P-T$ plane (Fig. $4 \mathrm{a}$ ). On the other hand, increasing $k_{1}$ results in a contraction of the SR along $P$ with a rotation of the main axis toward a zero slope in the $P-T$ plane (Fig. $4 \mathrm{~b}$ ).

These effects can be understood observing that the free energy of the system has a term $-k_{1} P^{2} N_{\mathrm{HB}}{ }^{(\Phi)}$. This term is associated to the fact 
(a)

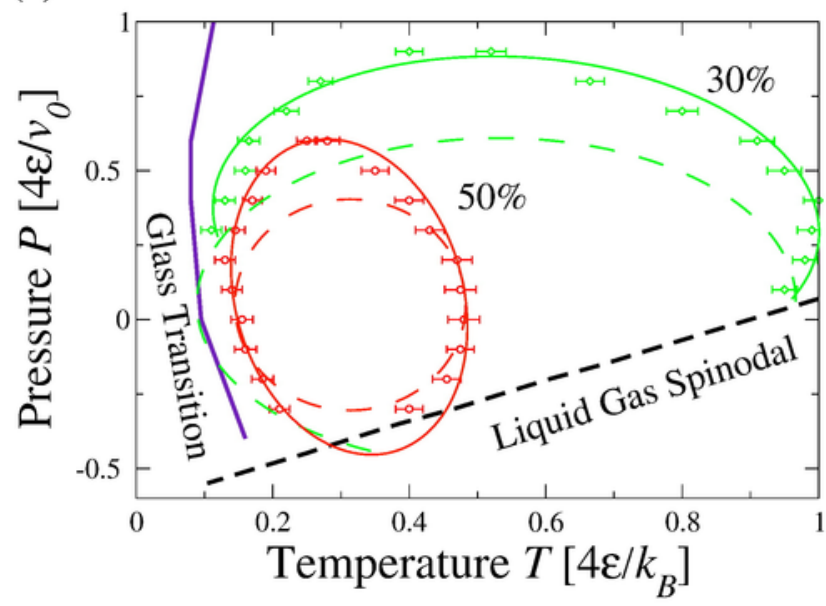

(b)

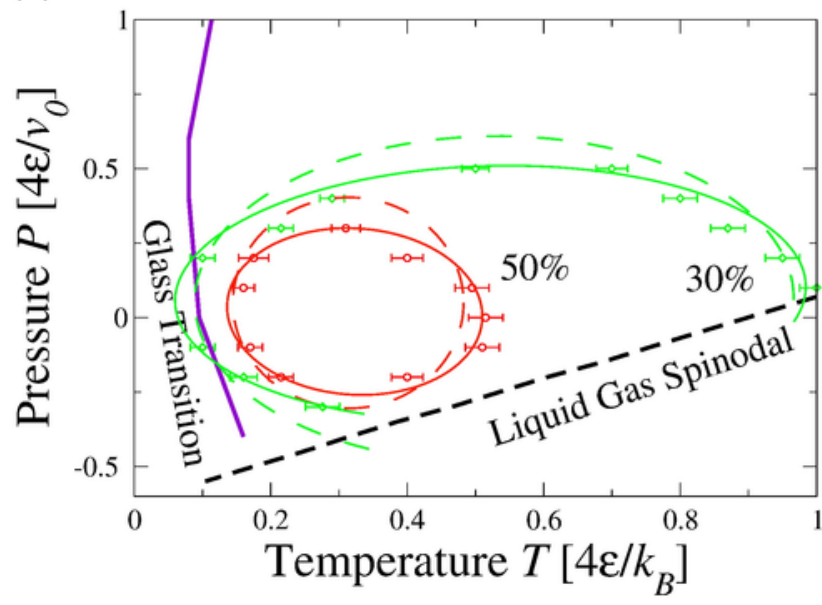

Fig. 4. Effect on the SR of changing the water compressibility factor $k_{1}$ at the $\Phi$-interface. Symbols and lines are as in Fig. 3 and the reference system has $k_{1} 4 \epsilon / v_{0}=1$. (a) For $k_{1} 4 \epsilon / v_{0}=0.5$, smaller than the reference value, the SR expands to a wider range of $P$ and the main ellipsis axis acquires a negative slope in the $P$ - $T$ plane. (b) For $k_{1} 4 \epsilon / v_{0}=1.5$, greater than the reference value, the SR contracts in $P$ and the main ellipsis axis becomes almost perpendicular to the $P$-axis. In both panels the effects of the change on the $T$-range of stability are minor.

that there is a larger water compressibility at the $\Phi$-interface, reducing the total free energy. Therefore, by decreasing $k_{1}$ the destabilizing effect of the increased water-compressibility is reduced and the protein gains stability in $P$ at constant $T$, while the opposite effect is achieved by increasing $k_{1}$. The observations about the slope of the contour lines discussed in the previous subsection apply also in this case explaining the rotation of the ellipsis axes.

\subsubsection{Varying the $H B$ volume-increase $v_{H B, 0}{ }^{(\Phi)}$ at the $\Phi$-interface and} $P=0$

A decrease of $v_{\mathrm{HB}, 0}{ }^{(\Phi)} / v_{0}$, with respect to the reference case, moves the SR at lower $P$, while an increase moves the SR at higher $P$ (Fig. 5). This effect can be understood observing that the free energy of the system has a term $P v_{\mathrm{HB}, 0}{ }^{(\Phi)} N_{\mathrm{HB}}{ }^{(\Phi)}$ that, at each $P$, implies a decreasing enthalpy cost for decreasing $v_{\mathrm{HB}, 0}{ }^{(\Phi)}$ if $N_{\mathrm{HB}}{ }^{(\Phi)}$ is kept constant. Hence, this term favors the unfolding at high $P$ when $v_{\mathrm{HB}, 0}(\Phi)$ is small, decreasing the stability of the native state upon pressurization (Fig. 5a). The opposite occurs for increasing $v_{\mathrm{HB}, 0}{ }^{(\Phi)}$ (Fig. 5b).

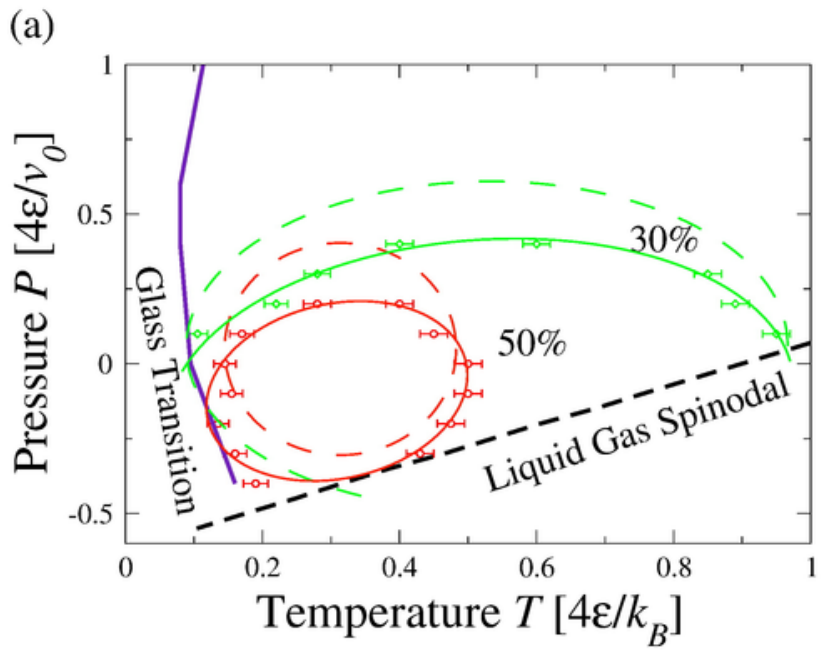

(b)

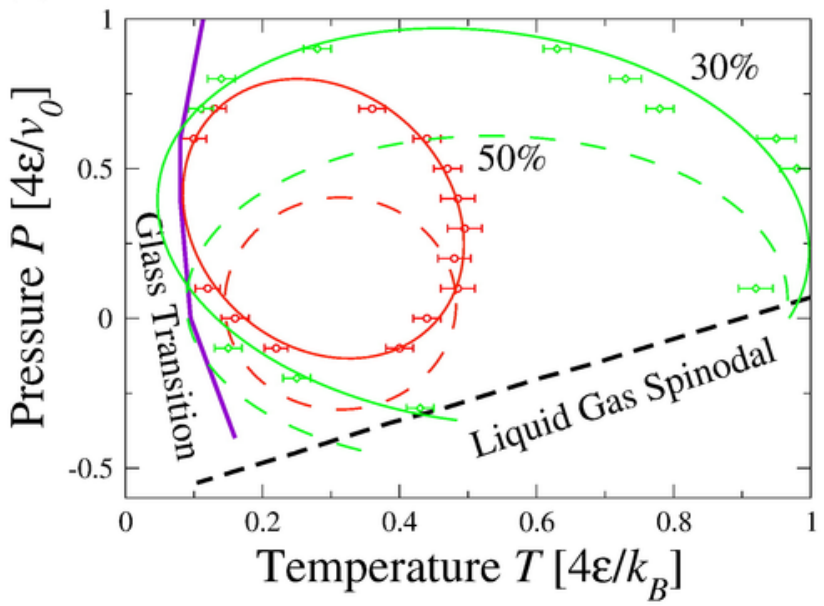

Fig. 5. Effect on the SR of changing the HB volume-increase $v_{\mathrm{HB}, 0}{ }^{(\Phi)}$ at the $\Phi$-interface and $P=0$. Symbols and lines are as in Fig. 3 and the reference system has $v_{\mathrm{HB}, 0}{ }^{(\Phi)} / v_{0}=0.5$. (a) For $v_{\mathrm{HB}, 0}{ }^{(\Phi)} / v_{0}=0.1$, smaller than the reference value, the SR moves toward lower $P$ and its main ellipsis axis rotates toward a positive slope in $P$ - $T$ plane. (b) For $v_{\mathrm{HB}, 0}{ }^{(\Phi)} / v_{0}=1$, greater than the reference value, the SR moves toward higher $P$ rotates toward a negative slope in $P-T$ plane. In both panels the effects of the change on the $T$-range of stability are minor.

We also find that the slope of the main ellipsis axis changes from positive, for small $v_{\mathrm{HB}, 0}{ }^{(\Phi)}$, to negative, for large $v_{\mathrm{HB}, 0}{ }^{(\Phi)}$. This is a consequence of the inversion of the contribution of the free-energy term $P v_{\mathrm{HB}, 0}{ }^{(\Phi)} N_{\mathrm{HB}}{ }^{(\Phi)}$ when $P$ changes sign. Because a variation of $v_{\mathrm{HB}, 0}{ }_{(\Phi)}$ changes where the SR crosses the $P=0$ axis, the stability contour-line changes shape as a consequence, resulting in an effective rotation of its elliptic main axis: the main axis is positive when the majority of the SR is at $P<0$ (Fig. 5a) and is negative otherwise (Fig. 5b).

3.1.4. Adding the quadratic P-dependence of $v_{H B}{ }^{(\Phi)}$ at the $\Phi$-interface So far we have shown the SRs for the model with $v_{\mathrm{HB}}{ }^{(\Phi)}$ linearly-dependent on $P$. This truncation of Eq. (6) implies that the model for $P<1 / k_{1} \equiv P_{L}$ describes a system where water-water HBs at the $\Phi$-interface decrease the local density, as expected, while for larger $P$ they do the opposite. Thanks to our specific choice of parameters for the reference system, our truncation does not affect the results because 
for $P>P_{L}$ the HB probability, both in bulk and at the $\Phi$-interface, is vanishing.

However, to check how qualitatively robust are our results against this truncation of Eq. (6), we consider also the case with the quadratic $P$-dependence of $v_{\mathrm{HB}}{ }^{(\Phi)}$, i.e.,

$$
v_{\mathrm{HB}}^{(\Phi)} / v_{\mathrm{HB}, 0}^{(\Phi)} \equiv 1-k_{1} P-k_{2} P^{2}
$$

where $k_{2}>0$ is a new parameter with units of $k_{1} / P$. With this new approximation of Eq. (6) results $P_{L} \equiv(2 / x)(\sqrt{1+x}-1)$, with $x \equiv$ $4 k_{2} / k_{1}$. Therefore, $P_{L}$ decreases for increasing $x$.

We fix $k_{1}$ to the reference value, and vary $k_{2}$ (Fig. 6). We find that for increasing $k_{2}$, the $\mathrm{SR}$ is progressively compressed on the high- $P$ side, with minor effects on the SR $T$-range. Adding a cubic term in Eq. (6) affects the SR in a similar way (data not shown). The rational for this behavior lies in the enhanced enthalpic gain upon exposing the $\Phi$-residue to the solvent since $v_{\mathrm{HB}}{ }^{(\Phi)}$ decreases faster upon approaching $P_{L}$ that, in turn, decreases for increasing $k_{2}$.

\subsubsection{Adding an attractive interaction $\epsilon_{w, \Phi}$ between water and $\Phi$ - residues}

Here, we check how a non-zero water-hydrophobic residue interaction, $\epsilon_{\mathrm{w}, \Phi}>0$, would affect the SR of the hydrophobic homopolymer. Indeed, despite the common misunderstanding of "water-phobia" due to the oversimplified terminology, it is well known that a hydrophobic interface attracts water, but with an interaction that is smaller than a hydrophilic surface.

We find that by setting $\epsilon_{\mathrm{w}, \Phi} / 4 \epsilon=0.05$, smaller than bulk water-water attraction, the SR is reduced in $P$ and lightly shifted toward lower $T$ (Fig. 7). In fact, an attractive water $-\Phi$ interaction enhances the propensity of the polymer to expose the $\Phi$ residues to the solvent, resulting in a global reduction of the SR and destabilizing the folded protein.

\subsubsection{Enhancing the cooperative interaction $J_{\sigma, \Phi}$ at the $\Phi$-interface}

Lastly, in the contest of the hydrophobic protein model, we consider a different scenario. As discussed in the model description, the



Fig. 6. Effect on the SR of adding the quadratic $P$-dependence of $v_{\mathrm{HB}}{ }^{(\Phi)}$ at the $\Phi$-interface. Symbols and lines are as in Fig. 3 and the reference system has $k_{1}=v_{0}^{2} / 4 \epsilon$ and $k_{2}=0$. For $k_{2}(4 \epsilon)^{2} / v_{0}^{3}=0.1$ (purple circles and line) with corresponding $P_{L} v_{0} \simeq 0.98$, $k_{2}(4 \epsilon)^{2} / v_{0}^{3}=0.5$ (not shown) with $P_{L} v_{0} \simeq 0.90$ and $k_{2}(4 \epsilon)^{2} / v_{0}^{3}=1$ (orange circles and line) with $P_{L} v_{0} \simeq 0.83$, the SR shrinks at high $P$ as $P_{L}$ decreases.

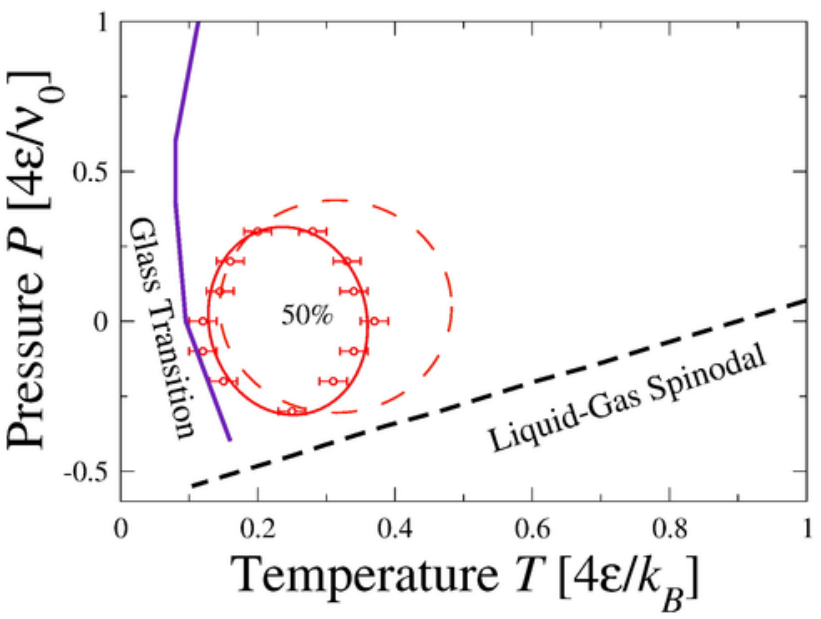

Fig. 7. Effect on the SR of adding an attractive interaction $\epsilon_{\mathrm{w}, \Phi}$ between water and $\Phi$-residues. Symbols and lines are as in Fig. 3 and the reference system has $\epsilon_{\mathrm{w}, \Phi}=0$. For $\epsilon_{\mathrm{w}, \Phi} / 4 \epsilon=0.05$, the SR moves toward lower $T$ and shrinks in $P$.

enthalpic gain upon cold denaturation would be consistent also with the assumption $J_{\sigma, \Phi}>J_{\sigma}$ associated to a larger cooperativity of the HBs at the $\Phi$-interface. Hence, to analyze this scenario, we compute the SR considering the directional component of the HB unaffected by the $\Phi$-interface $J_{\Phi}=J$, while assuming an enhanced HB cooperativity at the $\Phi$-interface $J_{\sigma, \Phi}>J_{\sigma}$. Note that the increase of $J_{\sigma, \Phi}$ promotes the number of cooperative HBs at the $\Phi$-interface only once they are formed as isolated HBs $\left(J_{\sigma, \Phi}<J_{\Phi}\right)$. Our finding (Fig. 8) is consistent with a close SR, presenting cold- and pressure-denaturation.

Although not discussed here, we expect that varying the parameters $k_{1}$ and $v_{\mathrm{HB}, 0}{ }^{(\Phi)}$, with the current choice of $J_{\sigma, \Phi}>J_{\sigma}$ and $J_{\Phi}=J$, would affect the SR similarly to the cases discussed in previous subsections.

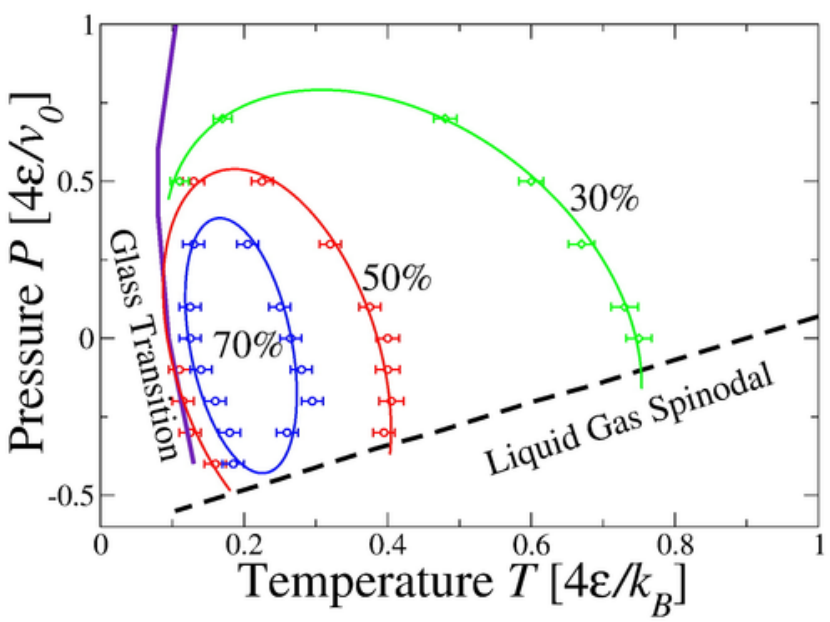

Fig. 8. Effect on the SR of enhancing the cooperative interaction $J_{\sigma \Phi}$ at the $\Phi$-interface. Symbols and lines are as in Fig. 3. Here we adopted $J_{\sigma, \Phi} / 4 \epsilon=0.1$, twice the value of $J_{\sigma}$ for bulk water molecules, while we fix $J_{\Phi} / 4 \epsilon=J / 4 \epsilon=0.3$. The parameters $k_{1}$ and $\nu_{\mathrm{HB}, 0}{ }^{(\Phi)}$ are as in Fig. $4 \mathrm{a}$. 


\subsection{Results for the polar protein model}

Next we summarize the results for the polar protein model. As shown in Ref. [21], also in this case the SR recovers a close elliptic-like SR in the $T-P$ plane (Fig. 9). In particular, despite that we reduce the value of $J_{\Phi} / 4 \epsilon$ with respect to the hydrophobic protein model in Table 1, the additional residue-residue interaction $\epsilon_{\mathrm{rr}}$ and water- $\zeta$-residue interaction $\epsilon_{\mathrm{w}, \zeta}$ stabilize the folded state to higher $P$ and $T$, as can been seen by comparing Fig. 9 with Fig. 2.

\subsubsection{Varying the residue-residue interaction $\epsilon_{r r}$}

To test how the residue-residue interaction $\epsilon_{\mathrm{rr}}$ is relevant for stabilizing the folded protein, we change its value. We find that an increase of $\epsilon_{\mathrm{rr}}$ results in a broadening of the SR in $T$ and $P$ (Fig. 10 a). We find the opposite effect if we reduce $\epsilon_{\text {rr }}$ (Fig. 10b). These results are consistent with our understanding that the native state is stabilized by stronger residue-residue interactions.

\subsubsection{Varying $J_{\Phi} / 4 \epsilon$ and $v_{H B, 0}{ }^{(\Phi)}$ at the $\Phi$-interface}

Next, we evaluate the effects of changing the water-water $J_{\Phi} / 4 \epsilon$ interaction and the $\mathrm{HB}$ volume increase constant $v_{\mathrm{HB}, 0}{ }^{(\Phi)}$ at the $\Phi$-interface for the polar protein model. We find that these changes affect

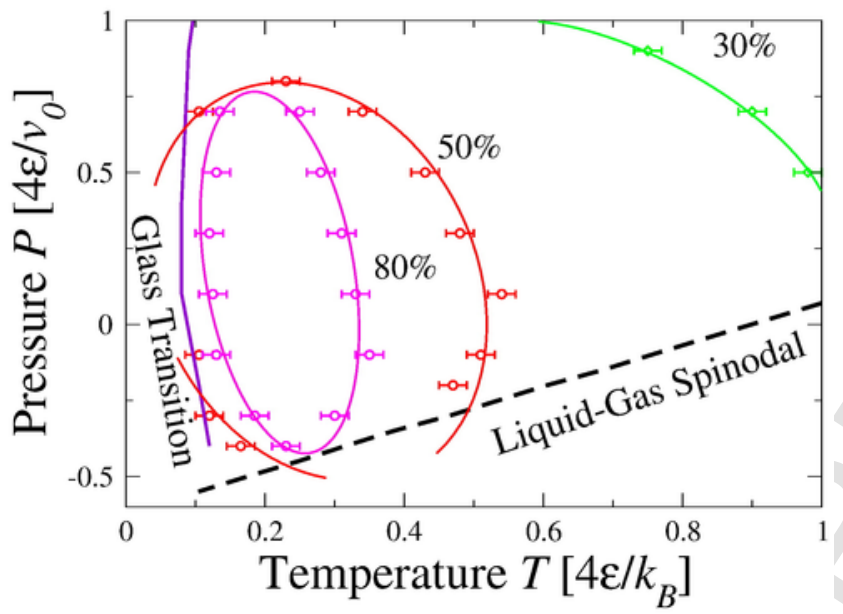

Fig. 9. The SR for the polar protein model. We set the parameters as in Table 2 with all the other parameters as in Table 1 . Symbols with continuous lines delimit the regions with $30 \%$ (green), $50 \%$ (red) and $80 \%$ (magenta) of the protein folded. The other lines are as in Fig. 2. All lines are guides for the eyes.

Table 1

Parameters for the reference system of the hydrophobic protein model (Fig. 2) with which we compare the results after varying the constants at the $\Phi$-interface one by one. We fix $v_{0} \equiv r_{0}^{3}=24.4 \AA^{3}$ and $\epsilon=5.8 \mathrm{~kJ} / \mathrm{mol}$.

\begin{tabular}{llllllll}
\hline$v_{\mathrm{HB}}^{(\mathrm{b})} / v_{0}$ & $J / 4 \epsilon$ & $J_{\sigma} / 4 \epsilon$ & $v_{\mathrm{HB}, 0}^{(\Phi)} / v_{0}$ & $J_{\Phi} / 4 \epsilon$ & $J_{\sigma, \Phi} / 4 \epsilon$ & $k_{1}(4 \epsilon) / v_{0}$ & $k_{2}=k_{3}$ \\
\hline 0.5 & 0.3 & 0.05 & 0.5 & 0.55 & 0.05 & 1 & 0 \\
\hline
\end{tabular}

Table 2

Additional parameters for the reference systems of the polar protein model (Fig. 9) with respect to those of the hydrophobic protein model in Table 1 . We also reduce the value of $J_{\Phi} / 4 \epsilon$ with respect to Table 1 .

\begin{tabular}{lllllll}
\hline Protein & $\epsilon_{\mathrm{rr}} / 4 \epsilon$ & $\epsilon_{\mathrm{w}, \Phi}$ & $\epsilon_{\mathrm{w}, \zeta} / 4 \epsilon$ & $J_{\Phi} / 4 \epsilon$ & $v_{\mathrm{HB}}{ }^{(\zeta)}$ & $J_{\zeta / 4 \epsilon}$ \\
\hline Polar & 0.2 & 0 & 0.35 & 0.5 & 0 & 0.4 \\
\hline
\end{tabular}

(a)

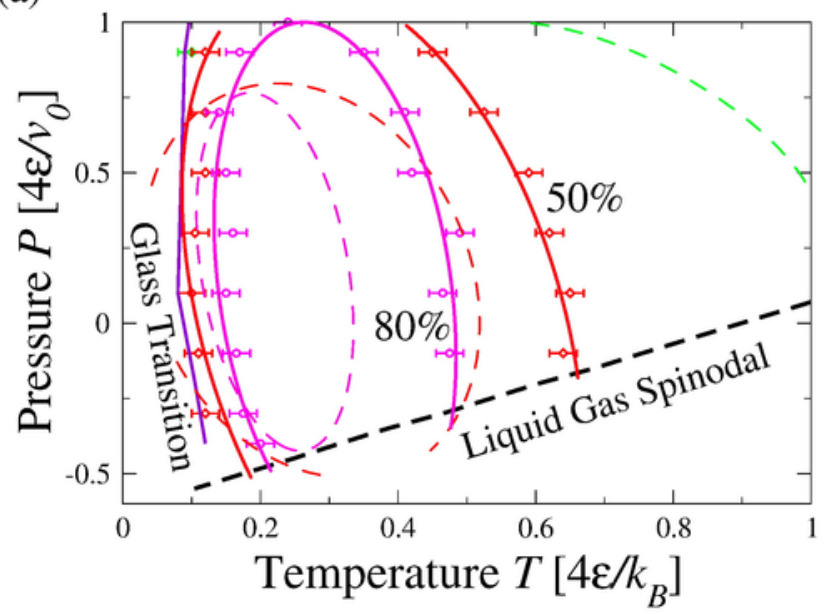

(b)

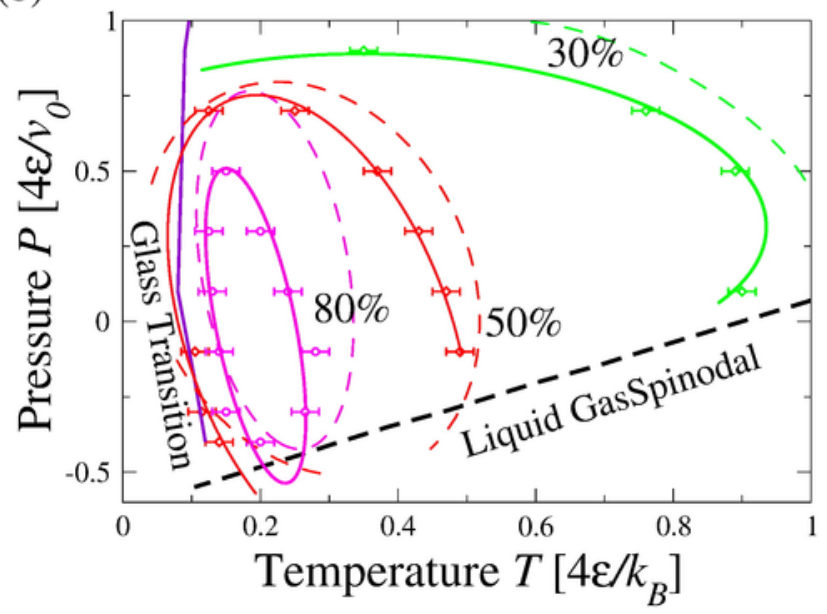

Fig. 10. Effect on the SR of the polar protein of varying the residue-residue interaction $\epsilon_{\mathrm{rr}}$. In both panels dashed lines are for the reference system in Fig. 9 (Table 2) with $\epsilon_{\mathrm{rr}} / 4 \epsilon=0.2$, continuous (with the same color code as for dashed lines) are for the systems with a modified $\epsilon_{\mathrm{rr}}$. (a) For $\epsilon_{\mathrm{rr}} / 4 \epsilon=0.5$, greater than the reference value, the SR expands in $P$ and $T$. (b) For $\epsilon_{\mathrm{rr}} / 4 \epsilon=0.05$, smaller than the reference value, the SR reduces in $P$ and $T$.

the SR in a fashion similar to those discussed for the hydrophobic protein model (not shown).

\section{Perspective on the protein design}

As we mentioned in the previous sections, the hydrated protein models discussed here simplify the dependence of the stability against unfolding on the protein sequence. In fact, in the homopolymer protein model, the sequence is reduced to a single amino acid, hence we have the alphabet $\mathscr{A}=1$, while in the polar protein model the alphabet size coincides, by construction, with the protein length $l, \mathscr{A}=l$, because the interaction matrix has $\left(l^{2}-l\right) / 2$ different elements that depend on the native state configuration.

In a more realistic case we would deal with proteins composed, at most, by 20 different amino acids, irrespective of the protein length. The amino acids assemble in a linear chain, which defines the protein sequence, in such a way that the protein is capable to fold into a unique native structure. Usually, among the huge amount of possible sequences, only few are good folders for a given native structure, 
smoothing and funneling the free energy landscape in order to lead the open protein conformation toward the native one.

Protein design strategies allow us to identify good folding sequences for each native conformation. Different methodologies have been proposed and studied in the past years [111-125] but water properties are not explicitly accounted, apart from a few cases $[111,114-116,120,122]$ usually referred only to ambient conditions. Despite the fact that the evolution has selected natural protein sequences capable to fold and work in extreme thermodynamic conditions (like the anti-freeze proteins or the thermophilic proteins), all the design methods are not efficient in establishing which are the key elements to predict artificial sequences stable in thermodynamic conditions far from the ambient situation.

On this important aspect our model can give a relevant insight. Indeed, following the works of Shakhnovich and Gutin [84,126] on lattice proteins, we can easily introduce an interaction matrix between the 20 amino acids - like the Miyazawa Jernigan residue-residue interaction matrix $S$ [127] — and look for the protein sequences which minimize the energy of the native structure. This scheme can be improved to account for the water properties of the surrounding water, since the protein interface affects the water-water hydrogen bonding at least in the first hydration shell. In this way, we aspect to find sequences with patterns depending on the $T$ and $P$ conditions of the surrounding water. Our preliminary results show that the protein sequences designed with our explicit-water model strongly depend on the thermodynamic conditions of the aqueous environment.

\section{Conclusions}

In this work we have presented a protein-water model to investigate the effect of the energy and density fluctuations at the hydrophobic interface $(\Phi)$ of the protein. In particular, we have considered two protein models. In the first we simplify the discussion assuming that the protein is a hydrophobic homopolymer. In the second model we consider a more realistic case, assuming that the protein has a


bic core and that the hydrophilic residues polarize the surrounding water molecules. These models can be considered as representative of the disordered proteins - where the collapsed protein state is not unique [1] - and of the ordered proteins, respectively. In both cases, we model the hydrophobic effects considering that the water-water hydrogen bond at the $\Phi$-interface is stronger with respect to the bulk, and that the corresponding density fluctuations are reduced upon pressurization.

Our model qualitatively reproduces the melting, the cold- and the pressure-denaturation experimentally observed in proteins. The stability region, i.e. the $T-P$ region where the protein attains its native state, has an elliptic-like shape in the $T-P$ plane, as predicted by the theory [42].

We discuss in detail how each interaction affects the stability region, showing that our findings are robust with respect to model parameters changes. Aiming at summarizing our findings, although the parameter variations results in a non-trivial modification of the protein stability region, we observe that the strength of the interfacial water-water HB compared to the bulk ones, mainly affects the $T$-stability range of proteins, while the compressibility of the hydrophobic hydration shell mainly regulates the $P$-stability range. The scenario remains substantially unvaried by changing the protein model from the oversimplified hydrophobic homopolymer to the polar protein model. Our findings put water's density and energy fluctuations in a primary role to maintain the stable protein structure and pave the way for a water-dependent design of artificial proteins, with tunable stability.

\section{Acknowledgments}

V.B. acknowledges the hospitality at the Universitat de Barcelona during his visits and the support from the Austrian Science Fund (FWF) project M 2150-N36. V.B. and I.C. acknowledge the support from the Austrian Science Fund (FWF) project P 26253-N27. G.F. acknowledges the support from the ICREA Academia prize, funded by the Generalitat de Catalunya, and from the FIS2015-66879-C2-2-P project, funded by the Spanish MINECO and the European Regional Development Fund (ERDF/FEDER). V.B., I.C. and G.F. acknowledge the support of the Erwin Schrödinger International Institute for Mathematics and Physics (ESI).

\section{References}

[1] D. Granata, F. Baftizadeh, J. Habchi, C. Galvagnion, A.D. Simone, C. Camil-loni, A. Laio, M. Vendruscolo, The inverted free energy landscape of an intrinsically disordered peptide by simulations and experiments, Sci. Rep. 5 (2015) 15449

[2] , Toward self-organization and complex matter, Science 295 (5564).

[3] G.M. Whitesides, B. Grzybowski, Self-assembly at all scales, Science 295 (5564).

[4] P. Yin, H.M.T. Choi, C.R. Calvert, N.A. Pierce, Programming biomolecular self-assembly pathways, Nature 451 (7176) (2008) 318-322 http://www.ncbi. nlm.nih.gov/pubmed/18202654https://doi.org/10.1038/nature06451.

[5] C.W. Carroll, A.F. Straight, Centromere formation: from epigenetics to self-assembly, Trends Cell Biol. 16 (2) (2006) 70-78, https://doi.org/10.1016/j.tcb. 2005.12.008.

[6] V. Bianco, A. Scialdone, M. Nicodemi, Colocalization of multiple DNA loci: a physical mechanism, Biophys. J. 103 (10) (2012) 2223-2232.

[7] S.M. Douglas, H. Dietz, T. Liedl, B. Högberg, F. Graf, W.M. Shih, Self-assembly of DNA into nanoscale three-dimensional shapes, Nature 459 (7245) (2009) 414-418 http://www.nature.com/doifinder/10.1038/nature08016https://doi.org/ 10.1038/nature08016.

[8] C.D. Michele, L. Rovigatti, T. Bellini, F. Sciortino, Self-assembly of short DNA duplexes: from a coarse-grained model to experiments through a theoretical link, Soft Matter 8 (32) (2012) 8388, https://doi.org/10.1039/c2sm25845e.

[9] M. Antonietti, S. Förster, Vesicles and liposomes: a self-assembly principle beyond lipids, Adv. Mater. 15 (16) (2003) 1323-1333, https://doi.org/10.1002/ adma.200300010.

[10] C. Calero, H.E. Stanley, G. Franzese, Structural interpretation of the large slowdown of water dynamics at stacked phospholipid membranes for decreasing hydration level: all-atom molecular dynamics, Materials 9 (5) (2016) 319 http://www.mdpi.com/1996-1944/9/5/319https://doi.org/10.3390/ma9050319.

[11] F. Nédélec, T. Surrey, E. Karsenti, Self-organisation and forces in the microtubule cytoskeleton, Curr. Opin. Cell Biol. 15 (1) (2003) 118-124, https://doi. org/10.1016/S0955-0674(02)00014-5.

[12] S. Gerecht-Nir, S. Cohen, J. Itskovitz-Eldor, Bioreactor cultivation enhances the efficiency of human embryoid body (hEB) formation and differentiation, Biotechnol. Bioeng. 86 (5) (2004) 493-502, https://doi.org/10.1002/bit.20045.

[13] K. Jakab, C. Norotte, B. Damon, F. Marga, A. Neagu, C.L. Besch-Williford, A. Kachurin, K.H. Church, H. Park, V. Mironov, R. Markwald, G. Vunjak-Novakovic, G. Forgacs, Tissue engineering by self-assembly of cells printed into topologically defined structures, 14 (3) (2017) https://doi.org/10.1089/TEN. 2007.0173.

[14] J. Rong, Z. Niu, L.A. Lee, Q. wang, Self-assembly of viral particles, Curr. Opin. Colloid Interface Sci. 16 (6) (2011) 441-450, https://doi.org/10.1016/j. cocis.2011.09.001.

[15] K.K. Sakimoto, C. Liu, J. Lim, P. Yang, Salt-induced self-assembly of bacteria on nanowire arrays, Nano Lett. 14 (9) (2014) 5471-5476 http://www.ncbi.nlm. nih.gov/pubmed/25115484https://doi.org/10.1021/nl502946j.

[16] E. van der Linden, P. Venema, Self-assembly and aggregation of proteins, Curr. Opin. Colloid Interface Sci. 12 (4) (2007) 158-165, https://doi.org/10.1016/j. cocis.2007.07.010.

[17] O. Vilanova, J.J. Mittag, P.M. Kelly, S. Milani, K.A. Dawson, J.O. Rädler, G. Franzese, Understanding the kinetics of protein-nanoparticle corona formation, ACS Nano 10 (12) (2016) 10842-10850, https://doi.org/10.1021/acsnano. 6b04858.

[18] C.M. Dobson, Protein folding and misfolding, Nature 426 (6968) (2003) 884-890 http://www.nature.com/doifinder/10.1038/nature02261https://doi.org/ 10.1038 /nature02261. 
[19] A. Finkelstein, O. Galzitskaya, Physics of protein folding, Phys. Life Rev. 1 (1) (2004) 23-56, https://doi.org/10.1016/j.plrev.2004.03.001.

[20] I. Coluzza, S.M. van der Vies, D. Frenkel, Translocation boost protein-folding efficiency of double-barreled chaperonins, Biophys. J. 90 (10) (2006) 3375-3381 http://www.ncbi.nlm.nih.gov/pubmed/16473898https://doi.org/10. 1529/biophysj.105.074898, http://www.pubmedcentral.nih.gov/articlerender. fcgi?artid=PMC1440723.

[21] V. Bianco, G. Franzese, Contribution of water to pressure and cold denaturation of proteins, Phys. Rev. Lett. 115 (10) (2015) 108101, https://doi.org/10.1103/ PhysRevLett.115.108101.

[22] S. Ghosh, S. Dey, M. Patel, R. Chakrabarti, Can an ammonium-based room temperature ionic liquid counteract the urea-induced denaturation of a small peptide?, Phys. Chem. Chem. Phys. 19 (2017) 7772-7787, https://doi.org/10 1039/C6CP08842B.

[23] Y. Levy, J.N. Onuchic, Water and proteins: a love-hate relationship., Proc. Natl. Acad. Sci. U.S.A. 101 (10) (2004) 3325-3326 http://www.pnas.org/ content/101/10/3325.fullhttps://doi.org/10.1073/pnas.0400157101.

[24] Y. Levy, J.N. Onuchic, Mechanisms of protein assembly: lessons from minimalist models, Accounts Chem. Res. 39 (2) (2006) 135-142 papers2:// publication/doi/10.1021/ar040204a.

[25] T.M. Raschke, Water structure and interactions with protein surfaces., Curr. Opin. Struct. Biol. 16 (2) (2006) 152-159 http://www.sciencedirect.com/ science/article/pii/S0959440X06000406https://doi.org/10.1016/j.sbi.2006.03. 002 .

[26] A. Zipp, W. Kauzmann, Pressure denaturation of metmyoglobin, Biochemistry 12 (21) (1973) 4217-4228, https://doi.org/10.1021/bi00745a028.

[27] P.L. Privalov, Cold denaturation of proteins, Crit. Rev. Biochem. Mol. Biol. 25 (4) (1990) 281-305.

[28] G. Hummer, S. Garde, A.E. Garcí, L.R. Pratt, The pressure dependence of hydrophobic interactions is consistent with the observed pressure denaturation of proteins, Proc. Natl. Acad. Sci. 95 (4) (1998) 1552-1555 http://www.pnas.org/ content/95/4/1552.abstract.

[29] F. Meersman, L. Smeller, K. Heremans, Pressure-assisted cold unfolding of proteins and its effects on the conformational stability compared to pressure and heat unfolding, High Pressure Res. 19 (1-6) (2000) 263-268 http://www. tandfonline.com/doi/abs/10.1080/08957950008202563https://doi.org/10.1080/ 08957950008202563.

[30] M.W. Lassalle, H. Yamada, K. Akasaka, The pressure-temperature free energy-landscape of staphylococcal nuclease monitored by (1)h NMR, J. Mol. Biol. 298 (2) (2000) 293-302 http://www.sciencedirect.com/science/article/pii/ S0022283600936593https://doi.org/10.1006/jmbi.2000.3659.

[31] L. Smeller, Pressure-temperature phase diagrams of biomolecules, Biochim. Biophys. Acta Protein Struct. Mol. Enzymol. 1595 (1-2) (2002) 11-29 http:// www.sciencedirect.com/science/article/B6T21-44XTS10-2/2/ 8ce4269f520742390b6b02395c0f6a35https://doi.org/10.1016/ S0167-4838(01)00332-6.

[32] H. Herberhold, R. Winter, Temperature- and pressure-induced unfolding and refolding of ubiquitin: a static and kinetic fourier transform infrared spectroscopy study, Biochemistry 41 (7) (2002) 2396-2401, https://doi.org/10.1021/ bi012023b.

[33] H. Lesch, H. Stadlbauer, J. Friedrich, J.M. Vanderkooi, Stability diagram and unfolding of a modified cytochrome $\mathrm{c}$ what happens in the transformation regime?, Biophys. J. 82 (3) (2002) 1644-1653 http://www.sciencedirect.com/ science/article/pii/S000634950275515Xhttps://doi.org/10.1016 S0006-3495(02)75515-X

[34] R. Ravindra, R. Winter, On the temperature-pressure free-energy landscape of proteins, Chem. Phys. Chem. 4 (4) (2003) 359-365 http://www.ncbi.nlm.nih. gov/pubmed/12728550https://doi.org/10.1002/cphc.200390062.

[35] F. Meersman, C.M. Dobson, K. Heremans, Protein unfolding, amyloid fibril formation and configurational energy landscapes under high pressure conditions., Chem. Soc. Rev. 35 (10) (2006) 908-917 http://pubs.rsc.org/en/Content/ ArticleHTML/2006/CS/B517761Hhttps://doi.org/10.1039/b517761h.

[36] A. Pastore, S.R. Martin, A. Politou, K.C. Kondapalli, T. Stemmler, P.A. Temussi, Unbiased cold denaturation: low- and high-temperature unfolding of yeast frataxin under physiological conditions, J. Am. Chem. Soc. 129 (17) (2007) 5374-5375, https://doi.org/10.1021/ja0714538.

[37] J. Wiedersich, S. Köhler, A. Skerra, J. Friedrich, Temperature and pressure dependence of protein stability: the engineered fluorescein-binding lipocalin flua shows an elliptic phase diagram, Proc. Natl. Acad. Sci. U.S.A. 105 (15) (2008) 5756-5761 http://www.pnas.org/content/105/15/5756.fullhttps://doi.org/10. 1073/pnas.0710409105.

[38] A. Maeno, H. Matsuo, K. Akasaka, The pressure-temperature phase diagram of hen lysozyme at low pH, Biophysics 5 (2009) 1-9 https://www.jstage.jst.go.jp/ article/biophysics/5/0/5_0_1/_articlehttps://doi.org/10.2142/biophysics.5.1.

[39] J. Somkuti, Z. Mártonfalvi, M.S.Z. Kellermayer, L. Smeller, Different pressure-temperature behavior of the structured and unstructured regions of titin, Biochim. Biophys. Acta 1834 (1) (2013) 112-118 http://www.sciencedirect.
com/science/article/pii/S1570963912002208https://doi.org/10.1016/j.bbapap. 2012.10 .001

[40] J. Somkuti, S. Jain, S. Ramachandran, L.á Smeller, Folding-unfolding transitions of Rv3221c on the pressure-temperature plane, High Pressure Res. 33 (2) (2013) 250-257 http://www.tandfonline.com/doi/abs/10.1080/08957959.2013. 780055https://doi.org/10.1080/08957959.2013.780055.

[41] N.V. Nucci, B. Fuglestad, E.A. Athanasoula, A.J. Wand, Role of cavities and hydration in the pressure unfolding of T4 lysozyme, Proc. Natl. Acad. Sci. U.S.A. 111 (38) (2014) 13846-13851 http://www.pnas.org/content/111/38/ 13846.fullhttps://doi.org/10.1073/pnas.1410655111.

[42] S.A. Hawley, Reversible pressure-temperature denaturation of chymotrypsinogen, Biochemistry 10 (13) (1971) 2436-2442 http://pubs.acs.org/doi/abs/10 1021/bi00789a002https://doi.org/10.1021/bi00789a002.

[43] Y.V. Griko, P.L. Privalov, J.M. Sturtevant, Cold denaturation of staphylococca nuclease., Proc. Natl. Acad. Sci. 85 (10) (1988) 3343-3347 http://www.pnas. org/content/85/10/3343.shorthttps://doi.org/10.1073/pnas.85.10.3343.

[44] K. Goossens, L. Smeller, J. Frank, K. Heremans, Pressure-tuning the conformation of bovine pancreatic trypsin inhibitor studied by fourier-transform infrared spectroscopy, Eur. J. Biochem. 236 (1) (1996) 254-262, https://doi.org/10. $1111 / j .1432-1033.1996 .00254 . x$

[45] D.P. Nash, J. Jonas, Structure of the pressure-assisted cold denatured state of ubiquitin, Biochem. Biophys. Res. Commun. 238 (2) (1997) 289-291.

[46] D.P. Nash, J. Jonas, Structure of pressure-assisted cold denatured lysozyme and comparison with lysozyme folding intermediates, Biochemistry 36 (47) (1997) 14375-14383 http://pubs.acs.org/doi/abs/10.1021/bi970881vhttps://doi.org/10. 1021/bi970881v.

[47] D. Paschek, A.E. García, Reversible temperature and pressure denaturation of a protein fragment: a replica exchange molecular dynamics simulation study, Phys. Rev. Lett. 93 (23) (2004) 238105 http://journals.aps.org/prl/abstract/10 1103/PhysRevLett.93.238105https://doi.org/10.1103/PhysRevLett.93.238105.

[48] J. Roche, J.A. Caro, D.R. Norberto, P. Barthe, C. Roumestand, J.L. Schlessman, A.E. Garcia, B.E. García-Moreno, C.A. Royer, A.E. García, B.E. Garcia-Moreno, C.A. Royer, Cavities determine the pressure unfolding of proteins, Proc. Natl. Acad. Sci. U.S.A. 109 (18) (2012) 6945-6950 http://www. pubmedcentral.nih.gov/articlerender.fcgi?artid $=3344970 \&$ tool=pmcentrez $\&$ rendertype=abstracthttps://doi.org/10.1073/pnas.1200915109, http://www.pnas. org/content/109/18/6945.

[49] E. Larios, M. Gruebele, Protein stability at negative pressure, Methods (San Diego, CA) 52 (1) (2010) 51-56 http://www.sciencedirect.com/science/article/ pii/S1046202310001283https://doi.org/10.1016/j.ymeth.2010.04.010.

[50] H.W. Hatch, F.H. Stillinger, P.G. Debenedetti, Computational study of the stability of the miniprotein trp-cage, the GB1 $\beta$-hairpin, and the AK16 peptide, under negative pressure, J. Phys. Chem. B 118 (28) (2014) 7761-7769, https://doi. org $/ 10.1021 / \mathrm{jp} 410651 \mathrm{u}$.

[51] P. De Los Rios, G. Caldarelli, Putting proteins back into water, Phys. Rev. E. 62 (6) (2000) 8449-8452, https://doi.org/10.1103/PhysRevE.62.8449.

[52] M.I. Marqués, J.M. Borreguero, H.E. Stanley, N.V. Dokholyan, Possible mechanism for cold denaturation of proteins at high pressure, Phys. Rev. Lett. 91 (13) (2003) 138103, https://doi.org/10.1103/PhysRevLett.91.138103.

[53] B.A. Patel, P.G. Debenedetti, F.H. Stillinger, P.J. Rossky, A water-explicit lattice model of heat-, cold-, and pressure-induced protein unfolding, Biophys. J. 93 (12) (2007) 4116-4127 http://www.sciencedirect.com/science/article/pii/ S0006349507716661https://doi.org/10.1529/biophysj.107.108530.

[54] M.V. Athawale, G. Goel, T. Ghosh, T.M. Truskett, S. Garde, Effects of lengthscales and attractions on the collapse of hydrophobic polymers in water, Proc. Natl. Acad. Sci. U.S.A. 104 (3) (2007) 733-738 http://www.pnas.org/content/ 104/3/733.fullhttps://doi.org/10.1073/pnas.0605139104.

[55] D. Nettels, S. Müller-Späth, F. Küster, H. Hofmann, D. Haenni, S. Rüegger, L. Reymond, A. Hoffmann, J. Kubelka, B. Heinz, K. Gast, R.B. Best, B. Schuler, Single-molecule spectroscopy of the temperature-induced collapse of unfolded proteins, Proc. Natl. Acad. Sci. 106 (49) (2009) 20740-20745 http://www.pnas. org/content/106/49/20740.abstracthttps://doi.org/10.1073/pnas.0900622106.

[56] R.B. Best, J. Mittal, Protein simulations with an optimized water model: cooperative helix formation and temperature-induced unfolded state collapse, J. Phys. Chem. B 114 (46) (2010) 14916-14923, https://doi.org/10.1021/ jp108618d.

[57] S.N. Jamadagni, C. Bosoy, S. Garde, Designing heteropolymers to fold into unique structures via water-mediated interactions, J. Phys. Chem. B 114 (42) (2010) 13282-13288, https://doi.org/10.1021/jp104924g.

[58] A.V. Badasyan, S.A. Tonoyan, Y.S. Mamasakhlisov, A. Giacometti, A.S. Benight, V.F. Morozov, Competition for hydrogen-bond formation in the helix-coil transition and protein folding, Phys. Rev. E Stat. Nonlinear Soft Matter Phys. 83 (5 Pt 1) (2011) 051903 http://journals.aps.org/pre/abstract/10.1103/ PhysRevE.83.051903https://doi.org/10.1103/PhysRevE.83.051903.

[59] S. Matysiak, P.G. Debenedetti, P.J. Rossky, Role of hydrophobic hydration in protein stability: a 3D water-explicit protein model exhibiting cold and heat de- 
naturation, J. Phys. Chem. B 116 (28) (2012) 8095-8104 http://pubs.acs.org/ doi/abs/10.1021/jp3039175https://doi.org/10.1021/jp3039175.

[60] V. Bianco, S. Iskrov, G. Franzese, Understanding the role of hydrogen bonds in water dynamics and protein stability, J. Biol. Phys. 38 (1) (2012-01-01) 27-48, https://doi.org/10.1007/s10867-011-9235-7.

[61] D. Paschek, S. Gnanakaran, A.E. Garcia, Simulations of the pressure and temperature unfolding of an alpha-helical peptide, Proc. Natl. Acad. Sci. U.S.A. 102 (19) (2005) 6765-6770 http://www.pnas.org/content/102/19/6765. shorthttps://doi.org/10.1073/pnas.0408527102.

[62] T. Sumi, H. Sekino, Possible mechanism underlying high-pressure unfolding of proteins: formation of a short-period high-density hydration shell, Phys. Chem. Chem. Phys. 13 (35) (2011) 15829-15832 http://www.ncbi.nlm.nih.gov/ pubmed/21814668https://doi.org/10.1039/c1cp21347d.

[63] I. Coluzza, A coarse-grained approach to protein design: learning from design to understand folding, PloS one 6 (7) (2011) e20853 http://www. pubmedcentral.nih.gov/articlerender.fcgi?artid $=3128589 \backslash \% 7 \mathrm{~B} \backslash \& \backslash$ $\% 7$ Dtool=pmcentrez $\backslash \% 7 \mathrm{~B} \backslash \& \backslash \% 7$ Drendertype=abstracthttps://doi.org $/ 10.1371 /$ journal.pone.0020853.

[64] C.L. Dias, Unifying microscopic mechanism for pressure and cold denaturations of proteins, Phys. Rev. Lett. 109 (4) (2012) 048104 http://journals.aps. org/prl/abstract/10.1103/PhysRevLett.109.048104https://doi.org/10.1103/ PhysRevLett.109.048104.

[65] P. Das, S. Matysiak, Direct characterization of hydrophobic hydration during cold and pressure denaturation, J. Phys. Chem. B 116 (18) (2012) 5342-5348 http://pubs.acs.org/doi/abs/10.1021/jp211832chttps://doi.org/10.1021/ jp211832c.

[66] R. Sarma, S. Paul, Effect of pressure on the solution structure and hydrogen bond properties of aqueous N-methylacetamide, Chem. Phys. 407 (0) (2012) 115-123 http://www.sciencedirect.com/science/article/pii/ S0301010412003436https://doi.org/10.1016/j.chemphys.2012.09.014.

[67] G. Franzese, V. Bianco, Water at biological and inorganic interfaces, Food Biophys. 8 (3) (2013) 153-169.

[68] S. Abeln, M. Vendruscolo, C.M. Dobson, D. Frenkel, A. simple lattice model, that captures protein folding, aggregation and amyloid formation, PloS one 9 (1) (2014) e85185 http://journals.plos.org/plosone/article?id=10.1371/journal. pone.0085185https://doi.org/10.1371/journal.pone.0085185.

[69] C. Yang, S. Jang, Y. Pak, A fully atomistic computer simulation study of cold denaturation of a $\beta$-hairpin., Nat. Commun. 5 (2014) 5773 http://www.nature. com/ncomms/2014/141215/ncomms6773/abs/ncomms6773.htmlhttps://doi.org/ $10.1038 /$ ncomms6773.

[70] L. Nisius, S. Grzesiek, Key stabilizing elements of protein structure identified through pressure and temperature perturbation of its hydrogen bond network., Nat. Chem. 4 (9) (2012) 711-717, https://doi.org/10.1038/nchem.1396.

[71] E. van Dijk, P. Varilly, T.P. Knowles, D. Frenkel, S. Abeln, Consistent treatment of hydrophobicity in protein lattice models accounts for cold denaturation, Phys. Rev. Lett. 116 (7) (2016) 078101 http://link.aps.org/doi/10.1103/ PhysRevLett.116.078101https://doi.org/10.1103/PhysRevLett.116.078101.

[72] K. Stokely, M.G. Mazza, H.E. Stanley, G. Franzese, Effect of hydrogen bond cooperativity on the behavior of water, Proc. Natl. Acad. Sci. U.S.A. 107 (2010) 1301-1306.

[73] E.G. Strekalova, M.G. Mazza, H.E. Stanley, G. Franzese, Large decrease of fluctuations for supercooled water in hydrophobic nanoconfinement, Phys. Rev. Lett. 106 (2011) 145701.

[74] O. Vilanova, G. Franzese, Structural and Dynamical Properties of Nanoconfined Supercooled Water, Arxiv:110.22864.

[75] G. Franzese, V. Bianco, S. Iskrov, Water at interface with proteins, Food Biophys. 6 (2011) 186-198, https://doi.org/10.1007/s11483-010-9198-4.

[76] M.G. Mazza, K. Stokely, S.E. Pagnotta, F. Bruni, H.E. Stanley, G. Franzese, More than one dynamic crossover in protein hydration water, Proc. Natl. Acad. Sci. 108 (50) (2011) 19873-19878 http://www.pnas.org/content/108/50/19873. abstracthttps://doi.org/10.1073/pnas.1104299108.

[77] V. Bianco, O. Vilanova, G. Franzese, Polyamorphism and polymorphism of a confined water monolayer: liquid-liquid critical point, liquid-crystal and crystal-crystal phase transitions, In: Proceedings of Perspectives and Challenges in Statistical Physics and Complex Systems for the Next Decade: A Conference in Honor of Eugene Stanley and Liacir Lucen, 2013, pp. 126-149.

[78] F. de los Santos, G. Franzese, Understanding diffusion and density anomaly in a coarse-grained model for water confined between hydrophobic walls, J. Phys. Chem. B. doi:10.1021/jp206197t http://pubs.acs.org/doi/abs/10.1021/ jp206197t.

[79] V. Bianco, G. Franzese, Critical behavior of a water monolayer under hydrophobic confinement, Sci. Rep. 4 (2014) 4440 http://www.pubmedcentral. nih.gov/articlerender.fcgi?artid $=3975237 \&$ tool $=$ pmcentrez\& rendertype $=$ abstract.

[80] L.E. Coronas, V. Bianco, A. Zantop, G. Franzese, Liquid-liquid critical point in 3D many-body water model, ArXiv e-prints arXiv:1610.00419.
[81] V. Bianco, G. Franzese, C. Dellago, I. Coluzza, Role of water in the selection of stable proteins at ambient and extreme thermodynamic conditions, Phys. Rev. X 7 (Jun 2017) 021047.

[82] N. Go, H. Taketomi, Respective roles of short-range and long-range interactions in protein folding, Proc. Natl. Acad. Sci. U. S. A. 75 (2) (1978) 559-563 http://apps.isiknowledge.com/InboundService.do?Func=Frame $\backslash \% 7 \mathrm{~B} \backslash \& \backslash$ $\% 7$ pproduct $=$ WOS $\backslash \% 7 \mathrm{~B} \backslash \& \backslash \% 7 \mathrm{Daction}=$ retrieve $\backslash \% 7 \mathrm{~B} \backslash \& \backslash \% 7 \mathrm{DSrcApp}=$ Papers $\backslash$ $\% 7 \mathrm{~B} \backslash \& \backslash \% 7 \mathrm{DUT}=\mathrm{A} 1978 \mathrm{EQ} 77900006 \% 7 \mathrm{~B} \backslash \& \backslash \% 7 \mathrm{DSID}=\mathrm{X} 17 @$ 9LCNjK4MCk2D6m6 \%7B $\backslash \& \backslash \% 7 D I n i t=Y e s \backslash \% 7 B \backslash \& \backslash$ $\% 7 \mathrm{DSrcAuth}=$ mekentosj $\backslash \% 7 \mathrm{~B} \backslash \& \backslash \% 7 \mathrm{Dmode}=$ FullRecord $\backslash \% 7 \mathrm{~B} \backslash \& \backslash$ $\% 7$ DcustomersID=mekentosj $\backslash \% 7 \mathrm{~B} \backslash \& \backslash \% 7 \mathrm{DD}$ estFail=http://www.isinet.

[83] M.S. Cheung, L.L. Chavez, J.N. Onuchic, The energy landscape for protein folding and possible connections to function, Polymer 45 (2) (2004) 547-555 http://links.isiglobalnet2.com/gateway/Gateway.cgi?GWVersion=2\\%7B\&\ $\% 7 D S r c$ Auth=mekentosj $\backslash \% 7 \mathrm{~B} \& \backslash \% 7 \mathrm{DSrcApp}=$ Papers $\backslash \% 7 \mathrm{~B} \& \backslash$ $\% 7 D D e s t L i n k T y p e=F u l l R e c o r d ~ \ \% 7 B \& \backslash \% 7 D D e s t A p p=W O S \backslash \% 7 B \& \backslash$ $\% 7 D K e y U T=000188045400022$ papers2://publication/doi/10.1016/j.polymer. 2003.10.082

[84] E.I. Shakhnovich, A.M. Gutin, A new approach to the design of stable proteins, Protein Eng. 6 (8) (1993) 793-800 http://peds.oxfordjournals.org/content/6/8/ 793.abstracthttps://doi.org/10.1093/protein/6.8.793.

[85] I. Coluzza, Transferable coarse-grained potential for de novo protein folding and design, PloS one 9 (12) (2014) e112852 http://dx.plos.org/10.1371/journal. pone.0112852https://doi.org/10.1371/journal.pone.0112852, http://www.ncbi. nlm.nih.gov/pubmed/25436908.

[86] I. Coluzza, Constrained versus unconstrained folding free-energy landscapes, Mol. Phys. (July) (2015) 1-8 http://www.tandfonline.com/doi/full/10.1080/ 00268976.2015.1043031https://doi.org/10.1080/00268976.2015.1043031.

[87] T. Morawietz, A. Singraber, C. Dellago, J. Behler, How van der Waals interactions determine the unique properties of water, Proc. Natl. Acad. Sci. U.S.A. 113 (30) (2016) 8368-8373 http://www.ncbi.nlm.nih.gov/pubmed/ 27402761https://doi.org/10.1073/pnas.1602375113, http://www.pubmedcentral. nih.gov/articlerender.fcgi?artid=PMC4968748.

[88] A.K. Soper, The radial distribution functions of water and ice from 220 to $673 \mathrm{k}$ and at pressures up to $400 \mathrm{MPa}$, Chem. Phys. 258 (2-3) 121-137. URL http:// www.sciencedirect.com/science/article/B6TFM-4105N7F-3/2/945bd5a597b647cb22ad87fedd6139f0.

[89] J. Teixeira, M.-C. Bellissent-Funel, Dynamics of water studied by neutron scattering, J. Phys.: Condens. Matter 2 (S) URL http://stacks.iop.org/0953-8984/2/ $\mathrm{i}=\mathrm{S} / \mathrm{a}=011$.

[90] L. Hernández de la Peña, P.G. Kusalik, Temperature dependence of quantum effects in liquid water, J. Am. Chem. Soc. 127 (14) (2005) 5246-5251 http:// pubs.acs.org/doi/abs/10.1021/ja0424676https://doi.org/10.1021/ja0424676.

[91] A.K. Soper, M.A. Ricci, Structures of high-density and low-density water, Phys. Rev. Lett. 84 (13) (2000) 2881-2884.

[92] K.F. Lau, K.A. Dill, A lattice statistical mechanics model of the conformationa and sequence spaces of proteins, Macromolecules 22 (10) (1989) 3986-3997 http://pubs.acs.org/doi/abs/10.1021/ma00200a030https://doi.org/10.1021/ ma00200a030.

[93] G. Caldarelli, P. de Los Rios, Cold and warm denaturation of proteins, J. Biol Phys. 27 (2-3) (2001) 229-241, https://doi.org/10.1023/A:1013145009949.

[94] I. Coluzza, H.G. Muller, D. Frenkel, Designing refoldable model molecules, Phys. Rev. E. 68 (4 Pt 2) (2003) 46703 http://www.ncbi.nlm.nih.gov/pubmed/ 14683075https://doi.org/10.1103/PhysRevE.68.046703.

[95] C.L. Dias, T. Ala-Nissila, M. Karttunen, I. Vattulainen, M. Grant, Microscopic mechanism for cold denaturation, Phys. Rev. Lett. 100 (11) (2008) 118101-118104 http://link.aps.org/abstract/PRL/v100/e118101.

[96] C. Petersen, K.-J. Tielrooij, H.J. Bakker, Strong temperature dependence of water reorientation in hydrophobic hydration shells, J. Chem. Phys. 130 (21) (2009) 214511 http://www.ncbi.nlm.nih.gov/pubmed/19508080https://doi.org/ $10.1063 / 1.3142861$

[97] S. Sarupria, S. Garde, Quantifying water density fluctuations and compressibility of hydration shells of hydrophobic solutes and proteins, Phys. Rev. Lett. 103 (3) (2009) 37803 http://link.aps.org/doi/10.1103/PhysRevLett.103.037803https: //doi.org/10.1103/PhysRevLett.103.037803.

[98] Y.I. Tarasevich, State and structure of water in vicinity of hydrophobic surfaces, Colloid J. 73 (2) (2011) 257-266 http://link.springer.com/10.1134/ S1061933X11020141https://doi.org/10.1134/S1061933X11020141.

[99] J.G. Davis, K.P. Gierszal, P. Wang, D. Ben-Amotz, Water structural transformation at molecular hydrophobic interfaces, Nature 491 (7425) (2012) 582-585, https://doi.org/10.1038/nature11570.

[100] D. Laage, T. Elsaesser, J.T. Hynes, Water dynamics in the hydration shells of biomolecules, Chem. Rev. (2017) http://pubs.acs.org/doi/abs/10.1021/acs. chemrev.6b00765https://doi.org/10.1021/acs.chemrev.6b00765, acs.chemrev.6b00765. 
[101] J. Grdadolnik, F. Merzel, F. Avbelj, Origin of hydrophobicity and enhanced water hydrogen bond strength near purely hydrophobic solutes, Proc. Natl. Acad. Sci. 114 (2) (2017) 322-327 http://www.pnas.org/content/114/2/322.

[102] N. Muller, Search for a realistic view of hydrophobic effects, Acc. Chem. Res. 23 (1) (1990) 23-28, https://doi.org/10.1021/ar00169a005.

[103] E.G. Strekalova, J. Luo, H.E. Stanley, G. Franzese, S.V. Buldyrev, Confinement of anomalous liquids in nanoporous matrices, Phys. Rev. Lett. 109 (10) (2012) 105701.

[104] K. Lum, D. Chandler, J.D. Weeks, Hydrophobicity at small and large length scales, J. Phys. Chem. B 103 (22) (1999) 4570-4577 http://pubs.acs.org/doi/ abs/10.1021/jp984327m.

[105] D. Schwendel, T. Hayashi, R. Dahint, A. Pertsin, M. Grunze, R. Steitz, F. Schreiber, Interaction of water with self-assembled monolayers: neutron reflectivity measurements of the water density in the interface region, Langmuir 19 (6) (2003) 2284-2293 http://pubs.acs.org/doi/abs/10.1021/la026716khttps://doi. org/10.1021/la026716k.

[106] T.R. Jensen, M. Østergaard Jensen, N. Reitzel, K. Balashev, G.H. Peters, K. Kjaer, T. Bjørnholm, Water in contact with extended hydrophobic surfaces: direct evidence of weak dewetting, Phys. Rev. Lett. 90 (8) (2003) $86101 \mathrm{http} / / /$ link.aps.org/doi/10.1103/PhysRevLett.90.086101https://doi.org/10.1103/ PhysRevLett.90.086101.

[107] D.A. Doshi, E.B. Watkins, J.N. Israelachvili, J. Majewski, Reduced water density at hydrophobic surfaces: effect of dissolved gases, Proc. Natl. Acad. Sci. U.S.A. 102 (27) (2005) 9458-9462 http://www.pnas.org/content/102/27/9458. abstracthttps://doi.org/10.1073/pnas.0504034102.

[108] R. Godawat, S.N. Jamadagni, S. Garde, Characterizing hydrophobicity of interfaces by using cavity formation, solute binding, and water correlations, Proc. Natl. Acad. Sci. U.S.A. 106 (36) (2009) 15119-15124 http://www.pnas.org/ content/106/36/15119.fullhttps://doi.org/10.1073/pnas.0902778106.

[109] T. Ghosh, A.E. García, S. Garde, Molecular dynamics simulations of pressure effects on hydrophobic interactions, J. Am. Chem. Soc. 123 (44) (2001) 10997-11003, https://doi.org/10.1021/ja010446v.

[110] C.L. Dias, H.S. Chan, Pressure-dependent properties of elementary hydrophobic interactions: ramifications for activation properties of protein folding, J. Phys. Chem. B 118 (27) (2014) 7488-7509, https://doi.org/10.1021/jp501935f.

[111] J.R. Desjarlais, T.M. Handel, De novo design of the hydrophobic cores of proteins., Protein Sci. Publ. Protein Soc. 4 (10) (1995) 2006-2018 http://www. pubmedcentral.nih.gov/articlerender.fcgi?artid=2142989\\%7B\&\} $\% 7$ Dtool=pmcentrez $\backslash \% 7 \mathrm{~B} \& \backslash \% 7$ Drendertype=abstracthttps://doi.org/10.1002/ pro.5560041006.

[112] C.K. Smith, L. Regan, Guidelines for protein design: the energetics of beta sheet side chain interactions, Science (New York, N.Y.) 270 (5238) (1995) 980-982 http://www.ncbi.nlm.nih.gov/pubmed/7481801.

[113] P.B. Harbury, J.J. Plecs, B. Tidor, T. Alber, P.S. Kim, High-resolution protein design with backbone freedom, Science (New York, N.Y.) 282 (5393) (1998) 1462-1467 http://www.ncbi.nlm.nih.gov/pubmed/9822371.

[114] B.I. Dahiyat, De novo protein design: fully automated sequence selection, Science 278 (5335) (1997) 82-87 http://www.sciencemag.org/cgi/doi/10.1126/ science.278.5335.82https://doi.org/10.1126/science.278.5335.82.

[115] B.I. Dahiyat, S.L. Mayo, Probing the role of packing specificity in protein design., Proc. Natl. Acad. Sci. U.S.A. 94 (19) (1997) 10172-10177 http://www. pubmedcentral.nih.gov/articlerender.fcgi?artid $=23334 \backslash \% 7 \mathrm{~B} \& \backslash$ $\% 7$ Dtool=pmcentrez $\backslash \% 7 \mathrm{~B} \& \backslash \%$ rendertype=abstract, http://www.pubmedcentral. nih.gov/articlerender.fcgi?artid=23334\& tool=pmcentrez\&rendertype $=$ abstract.
[116] B. Kuhlman, G. Dantas, G.C. Ireton, G. Varani, B.L. Stoddard, D. Baker, Design of a novel globular protein fold with atomic-level accuracy, Science (New York, N.Y.) 302 (5649) (2003) 1364-1368 http://www.ncbi.nlm.nih.gov/ pubmed/14631033https://doi.org/10.1126/science.1089427.

[117] M.E.M. Noble, J.A. Endicott, L.N. Johnson, Protein kinase inhibitors: insight into drug design from structure, Science 303 (5665) (2004) 1800-1805 http:// www.ncbi.nlm.nih.gov/pubmed/15031492https://doi.org/10.1126/science. 1095920, http://www.sciencemag.org/cgi/doi/10.1126/science.1095920.

[118] A.L. Borovinskiy, A.Y. Grosberg, Design of toy proteins capable of rearranging conformations in a mechanical fashion, J. Chem. Phys. 118 (11) (2003) 5201 http://link.aip.org/link/JCPSA6/v118/i11/p5201/s1\\%7B\&/ \%7DAgg=doihttps://doi.org/10.1063/1.1545774.

[119] I.N. Berezovsky, K.B. Zeldovich, E.I. Shakhnovich, Positive and negative design in stability and thermal adaptation of natural proteins, PLoS Comput. Biol. 3 (3) (2007) e52 http://dx.plos.org/10.1371/journal.pcbi.0030052https://doi.org/ 10.1371/journal.pcbi.0030052.

[120] D. Rothlisberger, O. Khersonsky, A.M. Wollacott, L. Jiang, J. Dechancie, J. Betker, J.L. Gallaher, E.A. Althoff, A. Zanghellini, O. Dym, S. Albeck, K.N. Houk, D.S. Tawfik, D. Baker, Kemp elimination catalysts by computational enzyme design, Nature 453 (7192) (2008) papers2://publication/doi/10.1038/ nature $06879,190-\mathrm{U} 4$.

[121] R. Das, D. Baker, Macromolecular modeling with Rosetta, Annu. Rev. Biochem. 77 (2008) 363-382 papers2://publication/doi/10.1146/annurev. biochem.77.062906.171838.

[122] L. Jiang, E.A. Althoff, F.R. Clemente, L. Doyle, D. Rothlisberger, A. Zanghellini, J.L. Gallaher, J.L. Betker, F. Tanaka, C.F. Barbas, D. Hilvert, K.N. Houk, B.L. Stoddard, D. Baker, De novo computational design of retro-aldol enzymes, Science 319 (5868) (2008) 1387-1391 http://science.sciencemag.org/ content/319/5868/1387.abstracthttps://doi.org/10.1126/science.1152692.

[123] N.P. King, W. Sheffler, M.R. Sawaya, B.S. Vollmar, J.P. Sumida, I. André, T. Gonen, T.O. Yeates, D. Baker, Computational design of self-assembling protein nanomaterials with atomic level accuracy, Science (New York, N.Y.) 336 (6085) (2012) 1171-1174 http://www.ncbi.nlm.nih.gov/pubmed/ 22654060https://doi.org/10.1126/science.1219364.

[124] S.E. Boyken, Z. Chen, B. Groves, R.A. Langan, G. Oberdorfer, A. Ford, J.M. Gilmore, C. Xu, F. DiMaio, J.H. Pereira, B. Sankaran, G. Seelig, P.H. Zwart, D. Baker, De novo design of protein homo-oligomers with modular hydrogen-bond network-mediated specificity, Science 352 (6286) (2016) 680-687 http://science.sciencemag.org/content/352/6286/680.abstracthttps://doi.org/10. 1126/science.aad8865.

[125] T.M. Jacobs, B. Williams, T. Williams, X. Xu, A. Eletsky, J.F. Federizon, T. Szyperski, B. Kuhlman, Design of structurally distinct proteins using strategies inspired by evolution, Science 352 (6286) (2016) 687-690 http://science. sciencemag.org/content/352/6286/687.abstracthttps://doi.org/10.1126/science. aad8036.

[126] E.I. Shakhnovich, A.M. Gutin, Engineering of stable and fast-folding sequences of model proteins., Proc. Natl. Acad. Sci. U.S.A. 90 (15) (1993) 7195-7199, https://doi.org/10.1073/pnas.90.15.7195.

[127] S. Miyazawa, R. Jernigan, Estimation of effective interresidue contact energies from protein crystal-structures - quasi-chemical approximation, Macromolecules 18 (3) (1985) 534-552. 